\title{
Logarithmic interpolation methods and measure of non-compactness
}

\author{
Blanca F. Besoy and Fernando Cobos* \\ Departamento de Análisis Matemático y Matemática Aplicada, Facultad de Matemáticas, Universidad \\ Complutense de Madrid. Plaza de Ciencias 3, 28040 Madrid, Spain.
}

\begin{abstract}
We derive interpolation formulae for the measure of non-compactness of operators interpolated by logarithmic methods with $\theta=0,1$ between quasi-Banach spaces. Applications are given to operators between Lorentz-Zygmund spaces.

Keywords: Logarithmic interpolation methods, measure of non-compactness, compact operators, Lorentz-Zygmund spaces.

2010 MSC: Primary 46M35, 47H08; Secondary 46B70, 46E30.
\end{abstract}

\section{Introduction}

Interpolation theory is a consolidated branch in functional analysis with important applications in harmonic analysis, partial differential equations, operator theory and other areas in mathematics. See, for example, the books by Butzer and Berens [9], Bergh and Löfström [4], Triebel [45, 46], Bennett and Sharpley [2], Conness [21] or Amrein, Boutet de Monvel and Georgescu $[1]$.

The most familiar interpolation methods are the real method $\left(A_{0}, A_{1}\right)_{\theta, q}$ and the complex method $\left[A_{0}, A_{1}\right]_{\theta}$, introduced by Lions and Peetre [35] and Calderón [10], respectively. If we work with a couple of Lebesgue spaces $\left(L_{p_{0}}(R), L_{p_{1}}(R)\right)$ with $1 \leq p_{0} \neq p_{1} \leq \infty, 0<\theta<1$, $1 / p=(1-\theta) / p_{0}+\theta / p_{1}$ and $1 \leq q \leq \infty$, it turns out that $\left[L_{p_{0}}(R), L_{p_{1}}(R)\right]_{\theta}=L_{p}(R)$ (equal norms) and $\left(L_{p_{0}}(R), L_{p_{1}}(R)\right)_{\theta, q}=L_{p, q}(R)$ (equivalent norms), where $L_{p, q}(R)$ is a Lorentz space (see $[4,45])$.

Logarithmic perturbations of the real method $\left(A_{0}, A_{1}\right)_{\theta, q, \mathbb{A}}$ have been also studied in detail in the literature. See, for example, the papers by Gustavsson [31], Doktorskii [24], Evans and Opic [29], Evans, Opic and Pick [30], Cobos and Segurado [20], Cobos, Fernández-Cabrera and Martínez [15] and Besoy and Cobos [6]. The norm in $\left(A_{0}, A_{1}\right)_{\theta, q, \mathbb{A}}$ is

$$
\|a\|_{\left(A_{0}, A_{1}\right)_{\theta, q, \mathbb{A}}}=\left(\sum_{m=-\infty}^{\infty}\left[2^{-\theta m} \ell^{\mathbb{A}}\left(2^{m}\right) K\left(2^{m}, a\right)\right]^{q}\right)^{1 / q} .
$$

Here $K$ is the Peetre's K-functional, $\mathbb{A}=\left(\alpha_{0}, \alpha_{\infty}\right) \in \mathbb{R}^{2}, \ell(t)=1+|\log t|$ and $\ell^{\mathbb{A}}(t)=\ell^{\alpha_{0}}(t)$ if $0<t \leq 1, \ell^{\mathbb{A}}(t)=\ell^{\alpha_{\infty}}(t)$ if $1<t<\infty$. Now $\theta$ can take also the extreme values 0 and

\footnotetext{
${ }^{*}$ Corresponding author.

Emails: cobos@mat.ucm.es (F. Cobos), blanca.f.besoy@ucm.es (B.F.Besoy).
} 
1, producing spaces very close to $A_{0}$ and $A_{1}$, respectively. These extreme cases are the most important for us here.

Applying logarithmic methods to a couple of Lebesgue spaces, we obtain Lorentz-Zygmund spaces (see $[3,38,25])$. In particular, if we choose the parameters $p_{0}, p_{1}, \theta, p, q$ as above and $\alpha \in \mathbb{R}$, then we have that $\left(L_{p_{0}}(R), L_{p_{1}}(R)\right)_{\theta, q,(\alpha, \alpha)}=L_{p, q}(\log L)_{\alpha}(R)$ (see [39, Proposition $6.2 /(\mathrm{c})])$.

In 1960 Kranosel'skiî [34] proved that if $T$ is a linear operator which satisfies the assumptions of the Riesz-Thorin convexity theorem, i.e. restrictions $T: L_{p_{j}}(R) \rightarrow L_{q_{j}}(S)$ are bounded for $1 \leq p_{0}, q_{0}, p_{1}, q_{1} \leq \infty$, and in addition $q_{0}<\infty$ and $T: L_{p_{0}}(R) \rightarrow L_{q_{0}}(S)$ is compact, then $T$ : $L_{p}(R) \rightarrow L_{q}(S)$ is also compact provided that $1 / p=(1-\theta) / p_{0}+\theta / p_{1}, 1 / q=(1-\theta) / q_{0}+\theta / q_{1}$ and $0<\theta<1$. This result led to the investigation of interpolation properties of compact operators under abstract interpolation methods. The first results already appeared in the seminal papers by Lions and Peetre [35] and Calderón [10]. See [11] for details and other references. As for the real method, the final result was achieved in 1992 by Cwikel [23] and Cobos, Kühn and Schonbek [18]. The problem is still open for the complex method.

In 2014 Edmunds and Opic [28] established a limiting variant of Kranosel'skiǐ's theorem for finite measure spaces with the outcome that if $T: L_{p_{0}}(R) \rightarrow L_{q_{0}}(S)$ is compact and $T:$ $L_{p_{1}}(R) \rightarrow L_{q_{1}}(S)$ is bounded, then $T$ is also compact when acting between Lorentz-Zygmund spaces which are very close to $L_{p_{0}}(R)$ and $L_{q_{0}}(S)$. Abstract versions of the result by Edmunds and Opic in terms of logarithmic methods for $\theta=0,1$ have been obtained by Cobos, FernándezCabrera and Martínez [13] and Cobos and Segurado [20]. In the last paper it is shown that the limit variant of Kranosel'skiř's theorem also holds when the function spaces are defined on $\sigma$-finite measure spaces. More recently, Besoy [5] has shown that the theorem is still valid if the function spaces which are target of $T$ are quasi-Banach spaces, that is, it is allowed that $q_{0}, q_{1}, q$ take any positive values.

The aim of this paper is to complete the research of $[28,13,20,5]$ by estimating the measure of non-compactness of the operator $T$ acting between the Lorentz-Zygmund spaces in terms of the measure of non-compactness of the restriction of $T$ from $L_{p_{0}}(R)$ into $L_{q_{0}}(S)$.

The behaviour under interpolation of the measure of non-compactness is a problem that has been studied by a number of authors. The case of the real method was considered by Edmunds and Texeira [44] assuming some extra conditions in the target couple, and by Cobos, FernándezMartínez and Martínez [17] for arbitrary Banach couples. Results for extensions of the real method have been obtained by Cordeiro [22], Szwedek [41] and Cobos, Fernández-Cabrera and Martínez [12]. Other quantitative results are due to Edmunds and Netrusov [26, 27] and Szwedek $[42,43]$. The case of bilinear operators has also been recently investigated by Mastyło and Silva [36] and by the present authors [7].

As far as logarithmic methods for $\theta=1,0$, if the operator acts between Banach couples, then estimates for the measure of non-compactness of the interpolated operator have been derived by Cobos, Fernández-Cabrera and Martínez [14, 15] with the help of the connection between the measure of non-compactness and another ideal measure (see [40]). We work here with quasiBanach couples where those connections are not available. Hence, our arguments are more direct. Following ideas originated in the papers by Cobos and Peetre [19] and Cobos, Kühn and Schonbek [18], we split the operator with the help of certain projections associated to the vector-valued sequence spaces that arise with the construction of the logarithmic spaces. Then we proceed to estimate the measure of non-compactness of each piece. Sometimes it suffices with the interpolation theorem in combination with some other auxiliary results, but for two of the pieces we have to construct suitable $\varepsilon$-nets. In those cases we rely on compactness in $\mathbb{R}^{n}$ of certain subsets defined by using the parameters of the logarithmic method, an idea that has 
his origin in [17] and our previous paper [7].

\section{Logarithmic interpolation spaces}

Let $\left(A,\|\cdot\|_{A}\right)$ be a quasi-Banach space. We put $c_{A} \geq 1$ for the constant in the quasi-triangle inequality. According to $[33, \S 15.10]$ or $[32$, Proposition 1.c.5], there is another quasi-norm $|\|\cdot|\||$ on $A$ which is equivalent to $\|\cdot\|_{A}$ and such that $\left.\|\cdot \cdot\|\right|^{p}$ satisfies the triangle inequality. Here $0<p \leq 1$ is the number given by the equality $c_{A}=2^{1 / p-1}$. We say that $(A,|\|\cdot|\||)$ is a $p$-Banach space. If $0<r<p$, it is clear that $(A,\||\cdot|\|)$ is also an $r$-Banach space. Note also that if $A$ is a $p$-Banach space then it satisfies the quasi-triangle inequality with constant $2^{1 / p-1}$.

If $B$ is another quasi-Banach space, we write $A=B$ if $A$ and $B$ coincide in the set-theoretical sense and if they have equivalent quasi-norms.

By a ( $p$-Banach) quasi-Banach couple $\bar{A}=\left(A_{0}, A_{1}\right)$ we mean two ( $p$-Banach) quasi-Banach spaces $A_{0}, A_{1}$ which are continuously embedded in the same Hausdorff topological vector space. For $t>0$ and $a \in A_{0}+A_{1}$, let

$$
K(t, a)=K\left(t, a ; A_{0}, A_{1}\right)=\inf \left\{\left\|a_{0}\right\|_{A_{0}}+t\left\|a_{1}\right\|_{A_{1}}: a=a_{0}+a_{1}, a_{j} \in A_{j}\right\},
$$

and if $a \in A_{0} \cap A_{1}$ put

$$
J(t, a)=J\left(t, a ; A_{0}, A_{1}\right)=\max \left\{\|a\|_{A_{0}}, t\|a\|_{A_{1}}\right\} .
$$

Then $K(1, \cdot)$ is the quasi-norm of $A_{0}+A_{1}$ and $J(1, \cdot)$ the quasi-norm of $A_{0} \cap A_{1}$. If $\|\cdot\|_{A_{j}}$ is a p-norm for $j=0,1$, then we put

$$
K_{p}(t, a)=K_{p}\left(t, a ; A_{0}, A_{1}\right)=\inf \left\{\left(\left\|a_{0}\right\|_{A_{0}}^{p}+t^{p}\left\|a_{1}\right\|_{A_{1}}^{p}\right)^{1 / p}: a=a_{0}+a_{1}, a_{j} \in A_{j}\right\} .
$$

The functionals $K(t, \cdot)$ and $K_{p}(t, \cdot)$ are equivalent quasi-norms in $A_{0}+A_{1}$ with

$$
K(t, a) \leq K_{p}(t, a) \leq 2^{1 / p-1} K(t, a), a \in A_{0}+A_{1} .
$$

Moreover, $K_{p}(t, \cdot)$ and $J(t, \cdot)$ are also $p$-norms.

Put $\ell(t)=1+|\log t|$ and for $\mathbb{A}=\left(\alpha_{0}, \alpha_{\infty}\right) \in \mathbb{R}^{2}$ write

$$
\ell^{\mathbb{A}}(t)=\ell^{\left(\alpha_{0}, \alpha_{\infty}\right)}(t)= \begin{cases}\ell^{\alpha_{0}}(t) & \text { if } 0<t \leq 1 \\ \ell^{\alpha_{\infty}}(t) & \text { if } 1<t<\infty\end{cases}
$$

For $0 \leq \theta \leq 1,0<q \leq \infty$ and $\mathbb{A} \in \mathbb{R}^{2}$, the logarithmic interpolation space $\bar{A}_{\theta, q, \mathbb{A}}=$ $\left(A_{0}, A_{1}\right)_{\theta, q, \mathbb{A}}$ is formed by all those $a \in A_{0}+A_{1}$ which have a finite quasi-norm

$$
\|a\|_{\bar{A}_{\theta, q, \mathbb{A}}}=\|a\|_{\left(A_{0}, A_{1}\right)_{\theta, q, \mathbb{A}}}=\left(\sum_{m=-\infty}^{\infty}\left[2^{-\theta m} \ell^{\mathbb{A}}\left(2^{m}\right) K\left(2^{m}, a\right)\right]^{q}\right)^{1 / q}
$$

(the sum should be replaced by the supremum when $q=\infty$ ). See $[29,30,20,15,6]$. Note that if $\mathbb{A}=(0,0)$ and $0<\theta<1$, then we recover the classical real interpolation space $\left(A_{0}, A_{1}\right)_{\theta, q}$ (see $[4,45,2,8])$. If $0<\theta<1$ and $\mathbb{A} \neq(0,0)$, then $\left(A_{0}, A_{1}\right)_{\theta, q, \mathbb{A}}$ is a special case of the real method with function parameter (see $[31,39])$.

Observe that $K\left(t, a ; A_{0}, A_{1}\right)=t K\left(t^{-1}, a ; A_{1}, A_{0}\right)$. This yields that

$$
\left(A_{0}, A_{1}\right)_{0, q,\left(\alpha_{0}, \alpha_{\infty}\right)}=\left(A_{1}, A_{0}\right)_{1, q,\left(\alpha_{\infty}, \alpha_{0}\right)} \text { (equal quasi-norms). }
$$


In what follows, we focus on the case $\theta=1$. We assume that

$$
\begin{cases}\alpha_{0}+1 / q<0 & \text { if } 0<q<\infty \\ \alpha_{0} \leq 0 & \text { if } q=\infty\end{cases}
$$

Then $\left(A_{0}, A_{1}\right)_{1, q, \mathbb{A}} \neq\{0\}$ and $\left(A_{0}, A_{1}\right)_{1, q, \mathbb{A}}$ is an intermediate space with respect to $\bar{A}$, that is

$$
A_{0} \cap A_{1} \hookrightarrow\left(A_{0}, A_{1}\right)_{1, q, \mathbb{A}} \hookrightarrow A_{0}+A_{1}
$$

where $\hookrightarrow$ means continuous embedding (see [30, Theorem 2.2]).

Given a quasi-Banach space $A$ intermediate with respect to $\bar{A}$ we write $A^{\circ}$ for the closure of $A_{0} \cap A_{1}$ in $A$.

It follows from the fundamental lemma (see [4, Lemma 3.3.2]) that $a \in\left(A_{0}+A_{1}\right)^{\circ}$ if and only if $\min (1,1 / t) K(t, a) \rightarrow 0$ as $t \rightarrow 0$ and as $t \rightarrow \infty$. It is easy to check from this fact that if

$$
\begin{cases}\alpha_{\infty}+1 / q \geq 0 & \text { if } 0<q<\infty \\ \alpha_{\infty}>0 & \text { if } q=\infty\end{cases}
$$

then

$$
\left(A_{0}, A_{1}\right)_{1, q, \mathbb{A}} \subseteq\left(A_{0}+A_{1}\right)^{\circ} .
$$

The Gagliardo completion $A_{j}^{\sim}$ of $A_{j}$ is formed by all those $a \in A_{0}+A_{1}$ having a finite quasi-norm

$$
\|a\|_{A_{j}}=\sup \left\{t^{-j} K(t, a): t>0\right\}
$$

(see $[2,4])$. It turns out that $a \in A_{0}+A_{1}$ belongs to $A_{j}^{\sim}$ if and only if there is a sequence $\left(a_{n}\right) \subseteq A_{j}$ with $\sup _{n}\left\|a_{n}\right\|_{A_{j}}<\infty$ and $\lim _{n \rightarrow \infty}\left\|a-a_{n}\right\|_{A_{0}+A_{1}}=0$. Let

$$
\||| a \mid\|_{j}=\inf \left\{\sup _{n}\left\|a_{n}\right\|_{A_{j}}: \lim _{n \rightarrow \infty}\left\|a-a_{n}\right\|_{A_{0}+A_{1}}=0\right\} .
$$

It turns out that \|\|$a\left\|_{j} \leq\right\| a\left\|_{A_{j}^{\sim}} \leq \max \left\{c_{A_{0}}, c_{A_{1}}\right\}\right\||| a \|_{j}$ (see [2, Theorem V.1.4]).

Minor modifications in the arguments of [2, Theorem V.1.5] show that

$$
K\left(t, a ; A_{0}^{\sim}, A_{1}^{\sim}\right) \leq K\left(t, a ; A_{0}, A_{1}\right) \leq \max \left\{c_{A_{0}}, c_{A_{1}}\right\} K\left(t, a ; A_{0}^{\sim}, A_{1}^{\sim}\right) .
$$

The quasi-Banach couple $\bar{A}$ is called mutually closed if $A_{j}=A_{j}^{\sim}$ for $j=0,1$.

Let $\Gamma$ be a quasi-Banach space of real valued sequences with $\mathbb{Z}$ as index set. We assume that $\Gamma$ contains all the sequences with only finitely many non-zero coordinates and that whenever $\left(\eta_{m}\right) \in \Gamma$ and $\left|\xi_{m}\right| \leq\left|\eta_{m}\right|$ for each $m \in \mathbb{Z}$, then $\left(\xi_{m}\right) \in \Gamma$ and $\left\|\left(\xi_{m}\right)\right\|_{\Gamma} \leq\left\|\left(\eta_{m}\right)\right\|_{\Gamma}$. Moreover, we assume that $\Gamma \hookrightarrow \ell_{p}+\ell_{p}\left(2^{-m}\right)$. Here $0<p \leq 1, \ell_{p}$ is the space of $p$-summable sequences and $\ell_{p}\left(2^{-m}\right)$ is the weighted space, with $\left(\xi_{m}\right) \in \ell_{p}\left(2^{-m}\right)$ if and only if $\left(2^{-m} \xi_{m}\right) \in \ell_{p}$.

Let $0<q \leq \infty, \mathbb{A}=\left(\alpha_{0}, \alpha_{\infty}\right) \in \mathbb{R}^{2}$ satisfying (2.2) and $0<p \leq 1$. Then $\Lambda=$ $\left(\ell_{p}, \ell_{p}\left(2^{-m}\right)\right)_{1, q, \mathbb{A}}$ is an example of quasi-Banach sequence space as introduced above.

If $\bar{A}=\left(A_{0}, A_{1}\right)$ is a $p$-Banach couple, the $J$-space $\bar{A}_{\Gamma ; J}=\left(A_{0}, A_{1}\right)_{\Gamma ; J}$ consists of all sums $a=\sum_{m=-\infty}^{\infty} u_{m}$ (convergence in $A_{0}+A_{1}$ ), where $\left(u_{m}\right) \subseteq A_{0} \cap A_{1}$ and $\left(J\left(2^{m}, u_{m}\right)\right) \in \Gamma$. The quasi-norm on $\bar{A}_{\Gamma ; J}$ is

$$
\|a\|_{\bar{A}_{\Gamma ; J}}=\|a\|_{\left(A_{0}, A_{1}\right)_{\Gamma ; J}}=\inf \left\{\left\|\left(J\left(2^{m}, u_{m}\right)\right)\right\|_{\Gamma}: a=\sum_{m=-\infty}^{\infty} u_{m}\right\}
$$

(see [37]).

The following result is a consequence of (2.4) and [37, Theorem 3.19]. For completeness we include a proof. 
Theorem 2.1. Let $\bar{A}=\left(A_{0}, A_{1}\right)$ be a mutually closed $p$-Banach couple $(0<p \leq 1)$. Let $0<q \leq \infty, \mathbb{A}=\left(\alpha_{0}, \alpha_{\infty}\right) \in \mathbb{R}^{2}$ satisfying (2.2) and (2.3) and put $\Lambda=\left(\ell_{p}, \ell_{p}\left(2^{-m}\right)\right)_{1, q, \mathbb{A}}$. Then we have with equivalent quasi-norms

$$
\left(A_{0}, A_{1}\right)_{1, q, \mathbb{A}}=\left(A_{0}, A_{1}\right)_{\Lambda ; J} .
$$

Proof. Take any $a \in\left(A_{0}, A_{1}\right)_{\Lambda ; J}$ and let $a=\sum_{m=-\infty}^{\infty} u_{m}$ be a $J$-representation of $a$ with $\left\|\left(J\left(2^{m}, u_{m}\right)\right)\right\|_{\Lambda} \leq 2\|a\|_{\left(A_{0}, A_{1}\right)_{\Lambda ; J}}$. For any $m \in \mathbb{Z}$, we have

$$
K_{p}\left(2^{k}, a\right) \leq\left(\sum_{m=-\infty}^{\infty} \min \left(1,2^{k-m}\right)^{p} J\left(2^{m}, u_{m}\right)^{p}\right)^{1 / p}
$$

Hence

$$
\begin{aligned}
\|a\|_{\left(A_{0}, A_{1}\right)_{1, q, \mathbb{A}}} & \leq\left(\sum_{k=-\infty}^{\infty}\left[2^{-k} \ell^{\mathbb{A}}\left(2^{k}\right)\left(\sum_{m=-\infty}^{\infty} \min \left(1,2^{k-m}\right)^{p} J\left(2^{m}, u_{m}\right)^{p}\right)^{1 / p}\right]^{q}\right)^{1 / q} \\
& \leq 2^{1 / p-1}\left\|\left(J\left(2^{m}, u_{m}\right)\right)\right\|_{\left(\ell_{p}, \ell_{p}\left(2^{-m}\right)\right)_{1, q, \mathbb{A}} \leq 2^{1 / p}\|a\|_{\left(A_{0}, A_{1}\right)_{\Lambda ; J}} .}
\end{aligned}
$$

Conversely, let $a \in\left(A_{0}, A_{1}\right)_{1, q, \mathbb{A}}$. Using (2.4) and [37, Theorem 3.2], we can find $\left(u_{m}\right) \subseteq A_{0} \cap A_{1}$ with $a=\sum_{m=-\infty}^{\infty} u_{m}$ (in $A_{0}+A_{1}$ ) and such that

$$
\left(\sum_{m=-\infty}^{\infty} \min \left(1,2^{k-m}\right)^{p} J\left(2^{m}, u_{m}\right)^{p}\right)^{1 / p} \leq c K\left(2^{k}, a\right), k \in \mathbb{Z},
$$

where $c=c_{p}$ is a constant independent of $a$ and $k$. Therefore

$$
\begin{aligned}
\|a\|_{\Lambda ; J} & \leq\left\|\left(J\left(2^{m}, u_{m}\right)\right)\right\|_{\left(\ell_{p}, \ell_{p}\left(2^{-m}\right)\right)_{1, q, \mathbb{A}}} \\
& \leq\left(\sum_{k=-\infty}^{\infty}\left[2^{-k} \ell^{\mathbb{A}}\left(2^{k}\right)\left(\sum_{m=-\infty}^{\infty} \min \left(1,2^{k-m}\right)^{p} J\left(2^{m}, u_{m}\right)^{p}\right)^{1 / p}\right]^{q}\right)^{1 / q} \\
& \leq c\left(\sum_{k=-\infty}^{\infty}\left[2^{-k} \ell^{\mathbb{A}}\left(2^{k}\right) K\left(2^{k}, a\right)\right]^{q}\right)^{1 / q}=c\|a\|_{\left(A_{0}, A_{1}\right)_{1, q, \mathbb{A}}}
\end{aligned}
$$

We complete this section with some results on the shift operator $\tau_{k}$ defined by $\tau_{k} \xi=\left(\xi_{m+k}\right)$ for $\xi=\left(\xi_{m}\right)$. Here $k \in \mathbb{Z}$.

Subsequently, given $\lambda \in \mathbb{R}$ we put $\lambda^{+}=\max \{0, \lambda\}$.

The following estimate is useful:

Let $\mathbb{A}=\left(\alpha_{0}, \alpha_{\infty}\right) \in \mathbb{R}^{2}$ and $\mathbb{B}=\left(\alpha_{0}^{+}+\left(-\alpha_{\infty}\right)^{+}, \alpha_{\infty}^{+}+\left(-\alpha_{0}\right)^{+}\right)$. Then for any $u>0$ we have

$$
\sup _{s>0} \frac{\ell^{\mathbb{A}}(s u)}{\ell^{\mathbb{A}}(s)} \leq \ell^{\mathbb{B}}(u) .
$$

Inequality (2.6) was established in [15, Lemma 2.1] for $u \geq 1$. If $0<u<1$ it follows by applying $[15$, Lemma 2.1] with $1 / u>1$. We obtain

$$
\sup _{s>0} \frac{\ell^{\mathbb{A}}(s u)}{\ell^{\mathbb{A}}(s)}=\sup _{s>0} \frac{\ell^{\left(\alpha_{\infty}, \alpha_{0}\right)}\left(\frac{1}{s} \frac{1}{u}\right)}{\ell^{\left(\alpha_{\infty}, \alpha_{0}\right)}\left(\frac{1}{s}\right)} \leq \ell^{\alpha_{0}^{+}+\left(-\alpha_{\infty}\right)^{+}}\left(\frac{1}{u}\right)=\ell^{\alpha_{0}^{+}+\left(-\alpha_{\infty}\right)^{+}}(u) .
$$


Lemma 2.2. Let $0<q \leq \infty$ and $\mathbb{A}=\left(\alpha_{0}, \alpha_{\infty}\right) \in \mathbb{R}^{2}$ satisfying (2.2). Put $\mathbb{B}=\left(\left(-\alpha_{\infty}\right)^{+}\right.$, $\alpha_{\infty}^{+}-$ $\left.\alpha_{0}\right)$. Then

$$
\left\|\tau_{k}\right\|_{\ell_{q}\left(2^{-m} \ell^{\mathbb{A}}\left(2^{m}\right)\right), \ell_{q}\left(2^{-m} \ell^{\mathbb{A}}\left(2^{m}\right)\right)} \leq 2^{k} \ell^{\mathbb{B}}\left(2^{-k}\right), \quad k \in \mathbb{Z} .
$$

Proof. For $\xi=\left(\xi_{m}\right)$ and $k \in \mathbb{Z}$, we have

$$
\begin{aligned}
\left\|\tau_{k} \xi\right\|_{\ell_{q}\left(2^{-m} \ell^{\mathbb{A}}\left(2^{m}\right)\right)} & =\left(\sum_{m=-\infty}^{\infty}\left(2^{-m} \ell^{\mathbb{A}}\left(2^{m}\right)\left|\xi_{m+k}\right|\right)^{q}\right)^{1 / q} \\
& \leq 2^{k} \sup _{m \in \mathbb{Z}}\left\{\frac{\ell^{\mathbb{A}}\left(2^{m}\right)}{\ell^{\mathbb{A}}\left(2^{m+k}\right)}\right\}\|\xi\|_{\ell_{q}\left(2^{-m} \ell^{\mathbb{A}}\left(2^{m}\right)\right)} \\
& \leq 2^{k} \ell^{\mathbb{B}}\left(2^{-k}\right)\|\xi\|_{\ell_{q}\left(2^{-m} \ell^{\mathbb{A}}\left(2^{m}\right)\right)}
\end{aligned}
$$

where we have used (2.6) in the last inequality.

Lemma 2.3. Let $0<q \leq \infty, \mathbb{A}=\left(\alpha_{0}, \alpha_{\infty}\right) \in \mathbb{R}^{2}$ satisfying (2.2), $\mathbb{B}=\left(\left(-\alpha_{\infty}\right)^{+}, \alpha_{\infty}^{+}-\alpha_{0}\right)$, $0<p \leq 1$ and $\Lambda=\left(\ell_{p}, \ell_{p}\left(2^{-m}\right)\right)_{1, q, \mathbb{A}}$. Then

$$
\left\|\tau_{k}\right\|_{\Lambda, \Lambda} \leq 2^{1 / p-1} 2^{k} \ell^{\mathbb{B}}\left(2^{-k}\right), \quad k \in \mathbb{Z} .
$$

Proof. Let $\xi=\left(\xi_{m}\right) \in \Lambda$. For any $r \in \mathbb{Z}$, we have

$$
\begin{aligned}
K\left(2^{r}, \xi ; \ell_{p}, \ell_{p}\left(2^{-m}\right)\right) & \leq K_{p}\left(2^{r}, \xi ; \ell_{p}, \ell_{p}\left(2^{-m}\right)\right) \\
& =\left(\sum_{m=-\infty}^{\infty}\left(\min \left(1,2^{r-m}\right)\left|\xi_{m}\right|\right)^{p}\right)^{1 / p} \leq 2^{1 / p-1} K\left(2^{r}, \xi ; \ell_{p}, \ell_{p}\left(2^{-m}\right)\right) .
\end{aligned}
$$

Hence, given any $k \in \mathbb{Z}$, we derive

$$
\begin{aligned}
\left\|\tau_{k} \xi\right\|_{\Lambda} & =\left(\sum_{r=-\infty}^{\infty}\left[2^{-r} \ell^{\mathbb{A}}\left(2^{r}\right) K\left(2^{r}, \xi ; \ell_{p}, \ell_{p}\left(2^{-m}\right)\right)\right]^{q}\right)^{1 / q} \\
& \leq\left(\sum_{r=-\infty}^{\infty}\left[2^{-r} \ell^{\mathbb{A}}\left(2^{r}\right)\left(\sum_{m=-\infty}^{\infty} \min \left(1,2^{r-m}\right)^{p}\left|\xi_{m+k}\right|^{p}\right)^{1 / p}\right]^{q}\right)^{1 / q} \\
& =\left(\sum_{r=-\infty}^{\infty}\left[2^{-r} \ell^{\mathbb{A}}\left(2^{r}\right)\left(\sum_{m=-\infty}^{\infty} \min \left(1,2^{r+k-m}\right)^{p}\left|\xi_{m}\right|^{p}\right)^{1 / p}\right]^{q}\right)^{1 / q} \\
& \leq 2^{1 / p-1} 2^{k} \sup _{r \in \mathbb{Z}}\left\{\frac{\ell^{\mathbb{A}}\left(2^{r}\right)}{\ell^{\mathbb{A}}\left(2^{r+k}\right)}\right\}\left(\sum_{r=-\infty}^{\infty}\left[2^{-(r+k)} \ell^{\mathbb{A}}\left(2^{r+k}\right) K\left(2^{r+k}, \xi ; \ell_{p}, \ell_{p}\left(2^{-m}\right)\right)\right]^{q}\right)^{1 / q} \\
& \leq 2^{1 / p-1} 2^{k} \ell^{\mathbb{B}}\left(2^{-k}\right)\|\xi\|_{\Lambda}
\end{aligned}
$$

where the last inequality follows from (2.6).

\section{Interpolation of the measure of non-compactness}

Let $\bar{A}=\left(A_{0}, A_{1}\right), \bar{B}=\left(B_{0}, B_{1}\right)$ be quasi-Banach couples. By $T \in \mathcal{L}(\bar{A}, \bar{B})$ we mean that $T$ is a bounded linear operator from $A_{0}+A_{1}$ into $B_{0}+B_{1}$ such that the restrictions $T: A_{j} \rightarrow B_{j}$ are bounded operators for $j=0,1$.

Given $0<q \leq \infty, \mathbb{A}=\left(\alpha_{0}, \alpha_{\infty}\right) \in \mathbb{R}^{2}$ satisfying (2.2) and $T \in \mathcal{L}(\bar{A}, \bar{B})$, it turns out that the restriction

$$
T:\left(A_{0}, A_{1}\right)_{1, q, \mathbb{A}} \rightarrow\left(B_{0}, B_{1}\right)_{1, q, \mathbb{A}}
$$


is bounded. Moreover, the argument given in [15, Theorem 2.2] for Banach couples can be adapted to our quasi-Banach setting with the effect that if $M_{j}>\|T\|_{A_{j}, B_{j}}$ for $j=0,1$, then

$$
\|T\|_{\bar{A}_{1, q, \mathbb{A}}, \bar{B}_{1, q, \mathbb{A}}} \leq 2 M_{1}\left(1+\left(\log \frac{M_{0}}{M_{1}}\right)^{+}\right)^{\alpha_{\infty}^{+}-\alpha_{0}} .
$$

In particular, if $\|T\|_{A_{1}, B_{1}}=0$ then we derive that $\|T\|_{\bar{A}_{1, q, \mathbb{A}}, \bar{B}_{1, q, \mathbb{A}}}=0$. Note also that if in addition to (2.2) we also have that (2.3) holds and $\|T\|_{A_{0}, B_{0}}=0$, then it follows from Theorem 2.1 that $\|T\|_{\bar{A}_{1, q, \mathbb{A}}, \bar{B}_{1, q, \mathbb{A}}}=0$.

Let $A, B$ be quasi-Banach spaces and $T \in \mathcal{L}(A, B)$. The operator $T$ is said to be compact if it transforms each bounded set of $A$ into a set whose closure is compact in $B$.

The (ball) measure of non-compactness $\beta(T)=\beta(T: A \rightarrow B)$ is defined to be the infimum of the set of all the numbers $\sigma>0$ for which there exists a finite subset $\left\{b_{1}, \ldots, b_{s}\right\} \subseteq B$ such that

$$
T\left(U_{A}\right) \subseteq \bigcup_{k=1}^{s}\left\{b_{k}+\varepsilon U_{B}\right\} .
$$

Here $U_{A}$ (respectively, $U_{B}$ ) is the closed unit ball of $A$ (respectively, $B$ ).

Clearly, $\beta(T) \leq\|T\|_{A, B}$. Moreover, $T$ is compact if and only if $\beta(T)=0$. The next two properties are easy to check and will be used freely in what follows:

(3.2) If $E$ is another quasi-Banach space and $S \in \mathcal{L}(B, E)$, then for $S T=S \circ T$ we have

$$
\beta(S T: A \rightarrow E) \leq\|S\|_{B, E} \beta(T: A \rightarrow B) .
$$

Moreover, if $\|S b\|_{E}=\|b\|_{B}$ for all $b \in B$, then $\beta(T: A \rightarrow B) \leq 2 c_{E} \beta(S T: A \rightarrow E)$.

(3.3) If $X$ is another quasi-Banach space and $R \in \mathcal{L}(X, A)$, then

$$
\beta(T R: X \rightarrow B) \leq\|R\|_{X, A} \beta(T: A \rightarrow B) .
$$

Moreover, if for any $a \in A$ with $\|a\|_{A}<1$, there is $x \in X$ with $\|x\|_{X}<1$ and $R x=a$, then $\beta(T: A \rightarrow B) \leq \beta(T R: X \rightarrow B)$.

Lemma 3.1. Let $\bar{A}=\left(A_{0}, A_{1}\right), \bar{B}=\left(B_{0}, B_{1}\right)$ be quasi-Banach couples and let $T \in \mathcal{L}(\bar{A}, \bar{B})$. Then

$$
\beta\left(T: A_{j}^{\sim} \rightarrow B_{j}^{\sim}\right) \leq \max \left\{c_{B_{0}}, c_{B_{1}}\right\} \beta\left(T: A_{j} \rightarrow B_{j}\right), j=0,1 .
$$

Proof. Suppose $j=0$ and let $\beta=\beta\left(T: A_{0} \rightarrow B_{0}\right)$. Given any $\varepsilon>0$ there is a finite set $\left\{b_{1}, \ldots, b_{s}\right\} \subseteq B_{0}$ such that

$$
T\left(U_{A_{0}}\right) \subseteq \bigcup_{k=1}^{s}\left\{b_{k}+(\beta+\varepsilon) U_{B_{0}}\right\} .
$$

Take any $a \in U_{A_{0}^{\sim}}$. There exists $\left(a_{n}\right) \subseteq A_{0}$ such that $\left\|a-a_{n}\right\|_{A_{0}+A_{1}} \rightarrow 0$ as $n \rightarrow \infty$ and $\left\|a_{n}\right\|_{A_{0}} \leq 1+\varepsilon$. Since

$$
\left(T a_{n}\right) \subseteq \bigcup_{k=1}^{s}\left\{(1+\varepsilon) b_{k}+(\beta+\varepsilon)(1+\varepsilon) U_{B_{0}}\right\},
$$

we can find $k_{0} \in\{1, \ldots, s\}$ and a subsequence $\left(T a_{n^{\prime}}\right)$ of $\left(T a_{n}\right)$ such that

$$
\left(T a_{n^{\prime}}\right) \subseteq\left\{(1+\varepsilon) b_{k_{0}}+(\beta+\varepsilon)(1+\varepsilon) U_{B_{0}}\right\} .
$$


It follows that

$$
\left\|T a_{n^{\prime}}-(1+\varepsilon) b_{k_{0}}\right\|_{B_{0}} \leq(\beta+\varepsilon)(1+\varepsilon), n^{\prime} \in \mathbb{N}
$$

and

$$
\begin{aligned}
\| T a- & (1+\varepsilon) b_{k_{0}}-\left(T a_{n^{\prime}}-(1+\varepsilon) b_{k_{0}}\right) \|_{B_{0}+B_{1}} \\
& \leq\|T\|_{A_{0}+A_{1}, B_{0}+B_{1}}\left\|a-a_{n^{\prime}}\right\|_{A_{0}+A_{1}} \rightarrow 0 \text { as } n^{\prime} \rightarrow \infty .
\end{aligned}
$$

Hence,

$$
\begin{aligned}
\left\|T a-(1+\varepsilon) b_{k_{0}}\right\|_{B_{0}^{\sim}} & \leq \max \left\{c_{B_{0}}, c_{B_{1}}\right\} \sup _{n^{\prime}}\left\{\left\|T a_{n^{\prime}}-(1+\varepsilon) b_{k_{0}}\right\|_{B_{0}}\right\} \\
& \leq \max \left\{c_{B_{0}}, c_{B_{1}}\right\}(\beta+\varepsilon)(1+\varepsilon) .
\end{aligned}
$$

This yields that

$$
T\left(U_{A_{0}}\right) \subseteq \bigcup_{k=1}^{s}\left\{(1+\varepsilon) b_{k}+(\beta+\varepsilon)(1+\varepsilon) \max \left\{c_{B_{0}}, c_{B_{1}}\right\} U_{B_{0}^{\sim}}\right\}
$$

and therefore $\beta\left(T: A_{0}^{\sim} \rightarrow B_{0}^{\sim}\right) \leq \max \left\{c_{B_{0}}, c_{B_{1}}\right\} \beta$.

The case $j=1$ can be treated similarly.

Subsequently we are going to work with vector-valued sequence spaces. Let $\left(W_{m}\right)$ be a sequence of quasi-Banach spaces with the same constant $c \geq 1$ in the quasi-triangle inequality for any $m \in \mathbb{Z}$, which is the case if $W_{m}$ is $p$-Banach for any $m \in \mathbb{Z}$. Let $\left(\lambda_{m}\right)$ be a sequence of positive numbers and $0<q \leq \infty$. We write

$$
\ell_{q}\left(\lambda_{m} W_{m}\right)=\left\{w=\left(w_{m}\right): w_{m} \in W_{m} \text { and }\|w\|_{\ell_{q}\left(\lambda_{m} W_{m}\right)}=\left\|\left(\lambda_{m}\left\|w_{m}\right\|_{W_{m}}\right)\right\|_{\ell_{q}}<\infty\right\} .
$$

If $\Gamma$ is a quasi-Banach sequence lattice, we define $\Gamma\left(\lambda_{m} W_{m}\right)$ in a similar way.

Lemma 3.2. Let $0<p \leq 1$ and let $\left(W_{m}\right)$ be a sequence of $p-$ Banach spaces. Assume that $0<q \leq \infty$ and let $\mathbb{A}=\left(\alpha_{0}, \alpha_{\infty}\right) \in \mathbb{R}^{2}$ satisfying (2.2). Let $\Lambda=\left(\ell_{p}, \ell_{p}\left(2^{-m}\right)\right)_{1, q, \mathbb{A}}$. We have with equivalence of quasi-norms

$$
\left(\ell_{p}\left(W_{m}\right), \ell_{p}\left(2^{-m} W_{m}\right)\right)_{1, q, \mathbb{A}}=\Lambda\left(W_{m}\right) .
$$

Proof. Let $w=\left(w_{m}\right) \in\left(\ell_{p}\left(W_{m}\right), \ell_{p}\left(2^{-m} W_{m}\right)\right)_{1, q, \mathbb{A}}$. For any $k \in \mathbb{Z}$ and $\varepsilon>0$, we can find $w^{j}=\left(w_{j, m}\right) \in \ell_{p}\left(2^{-j m} W_{m}\right)$ such that $w=w^{0}+w^{1}$ and $\left\|w^{0}\right\|_{\ell_{p}\left(W_{m}\right)}+2^{k}\left\|w^{1}\right\|_{\ell_{p}\left(2^{-m} W_{m}\right)} \leq$ $(1+\varepsilon) K\left(2^{k}, w ; \ell_{p}\left(W_{m}\right), \ell_{p}\left(2^{-m} W_{m}\right)\right)$. Hence,

$$
\begin{aligned}
\left(\sum_{m=-\infty}^{\infty} \min \left(1,2^{k-m}\right)^{p}\left\|w_{m}\right\|_{W_{m}}^{p}\right)^{1 / p} & \leq\left(\left\|w^{0}\right\|_{\ell_{p}\left(W_{m}\right)}^{p}+2^{k p}\left\|w^{1}\right\|_{\ell_{p}\left(2^{-m} W_{m}\right)}^{p}\right)^{1 / p} \\
& \leq 2^{1 / p-1}(1+\varepsilon) K\left(2^{k}, w ; \ell_{p}\left(W_{m}\right), \ell_{p}\left(2^{-m} W_{m}\right)\right) .
\end{aligned}
$$

It follows that

$$
\begin{aligned}
\|w\|_{\Lambda\left(W_{m}\right)} & =\left(\sum_{k=-\infty}^{\infty}\left[2^{-k} \ell^{\mathbb{A}}\left(2^{k}\right) K\left(2^{k},\left(\left\|w_{m}\right\|_{W_{m}}\right) ; \ell_{p}, \ell_{p}\left(2^{-m}\right)\right)\right]^{q}\right)^{1 / q} \\
& \leq\left(\sum_{k=-\infty}^{\infty}\left[2^{-k} \ell^{\mathbb{A}}\left(2^{k}\right)\left(\sum_{m=-\infty}^{\infty} \min \left(1,2^{k-m}\right)^{p}\left\|w_{m}\right\|_{W_{m}}^{p}\right)^{1 / p}\right]^{q}\right)^{1 / q} \\
& \leq 2^{1 / p-1}\left(\sum_{k=-\infty}^{\infty}\left[2^{-k} \ell^{\mathbb{A}}\left(2^{k}\right) K\left(2^{k}, w ; \ell_{p}\left(W_{m}\right), \ell_{p}\left(2^{-m} W_{m}\right)\right)\right]^{q}\right)^{1 / q} \\
& =2^{1 / p-1}\|w\|_{\left(\ell_{p}\left(W_{m}\right), \ell_{p}\left(2^{-m} W_{m}\right)\right)_{1, q, \mathbb{A}}} .
\end{aligned}
$$


Conversely, if $w=\left(w_{m}\right) \in \Lambda\left(W_{m}\right)$, given $k \in \mathbb{Z}$, put $w^{j}=\left(w_{j, m}\right)$ with

$$
w_{0, m}=\left\{\begin{array}{ll}
w_{m} & \text { if } m \leq k, \\
0 & \text { if } m>k,
\end{array} \quad w_{1, m}= \begin{cases}0 & \text { if } m \leq k, \\
w_{m} & \text { if } m>k .\end{cases}\right.
$$

Then $w=w^{0}+w^{1}$ and

$$
\begin{aligned}
K\left(2^{k}, w ; \ell_{p}\left(W_{m}\right), \ell_{p}\left(2^{-m} W_{m}\right)\right) & \leq\left(\sum_{m=-\infty}^{k}\left\|w_{m}\right\|_{W_{m}}^{p}\right)^{1 / p}+2^{k}\left(\sum_{m=k+1}^{\infty}\left(2^{-m}\left\|w_{m}\right\|_{W_{m}}\right)^{p}\right)^{1 / p} \\
& \leq 2\left(\sum_{m=-\infty}^{\infty} \min \left(1,2^{k-m}\right)^{p}\left\|w_{m}\right\|_{W_{m}}^{p}\right)^{1 / p}
\end{aligned}
$$

Therefore,

$$
\begin{aligned}
\|w\|_{\left(\ell_{p}\left(W_{m}\right), \ell_{p}\left(2^{-m} W_{m}\right)\right)_{1, q, \mathbb{A}}} & =\left(\sum_{k=-\infty}^{\infty}\left[2^{-k} \ell^{\mathbb{A}}\left(2^{k}\right) K\left(2^{k}, w ; \ell_{p}\left(W_{m}\right), \ell_{p}\left(2^{-m} W_{m}\right)\right)\right]^{q}\right)^{1 / q} \\
& \leq 2\left(\sum_{k=-\infty}^{\infty}\left[2^{-k} \ell^{\mathbb{A}}\left(2^{k}\right)\left(\sum_{m=-\infty}^{\infty} \min \left(1,2^{k-m}\right)^{p}\left\|w_{m}\right\|_{W_{m}}^{p}\right)^{1 / p}\right]^{q}\right)^{1 / q} \\
& \leq 2^{1 / p}\|w\|_{\Lambda\left(W_{m}\right)} .
\end{aligned}
$$

We also recall the following embedding (see [5, Lemma 3.5]).

Lemma 3.3. Let $\left(W_{m}\right)$ be a sequence of quasi-Banach spaces with the same constant $c \geq 1$ in the quasi-triangle inequality. Let $0<q \leq \infty$ and $\mathbb{A}=\left(\alpha_{0}, \alpha_{\infty}\right) \in \mathbb{R}^{2}$ satisfying (2.2). Then

$$
\left(\ell_{\infty}\left(W_{m}\right), \ell_{\infty}\left(2^{-m} W_{m}\right)\right)_{1, q, \mathbb{A}} \hookrightarrow \ell_{q}\left(2^{-m} \ell^{\mathbb{A}}\left(2^{m}\right) W_{m}\right) .
$$

Given $0<q \leq \infty, \mathbb{A} \in \mathbb{R}^{2}$ satisfying (2.2) and any quasi-Banach couple $\bar{B}=\left(B_{0}, B_{1}\right)$, there are natural vector valued sequence spaces that arise with the construction of $\left(B_{0}, B_{1}\right)_{1, q, \mathbb{A}}$. Indeed, let $F_{m}=\left(B_{0}+B_{1}, K\left(2^{m}, \cdot\right)\right), m \in \mathbb{Z}$. The sequence $\left(F_{m}\right)$ is formed by quasi-Banach spaces with the same constant $c=\max \left\{c_{B_{0}}, c_{B_{1}}\right\}$ in the quasi-triangle inequality. Consider the spaces $\ell_{q}\left(2^{-m} \ell^{\mathbb{A}}\left(2^{m}\right) F_{m}\right), \ell_{\infty}\left(F_{m}\right)$ and $\ell_{\infty}\left(2^{-m} F_{m}\right)$. The operator $\iota b=(\ldots, b, b, b, \ldots)$ is a metric injection from $\left(B_{0}, B_{1}\right)_{1, q, \mathbb{A}}$ into $\ell_{q}\left(2^{-m} \ell^{\mathbb{A}}\left(2^{m}\right) F_{m}\right)$. Note also that $\iota: B_{j} \rightarrow \ell_{\infty}\left(2^{-m j} F_{m}\right)$, $j=0,1$, is bounded with quasi-norm less than or equal to 1 .

We use this notation to state the next lemma which follows from a bilinear result of the present authors [7, Lemma 3.2].

Lemma 3.4. Let $\bar{A}=\left(A_{0}, A_{1}\right), \bar{B}=\left(B_{0}, B_{1}\right)$ be quasi-Banach couples and let $T \in \mathcal{L}(\bar{A}, \bar{B})$. Fix $j \in\{0,1\}$ and put $\beta_{j}=\beta\left(T: A_{j} \rightarrow B_{j}\right)$. Assume that there is a quasi-Banach space $X$ and bounded linear operators $R_{n} \in \mathcal{L}\left(X, A_{j}\right)$ such that $\left\|R_{n}\right\|_{X, A_{j}} \leq 1$ and $\lim _{n \rightarrow \infty}\left\|T R_{n}\right\|_{X, B_{0}+B_{1}}=$ 0. Then the following holds.

a) If $\beta_{j}=0$, then there is a subsequence $\left(n^{\prime}\right)$ such that $\lim _{n^{\prime} \rightarrow \infty}\left\|\iota T R_{n^{\prime}}\right\|_{X, \ell_{\infty}\left(2^{-m j} F_{m}\right)}=0$.

b) If $\beta_{j}>0$, then there is a constant $C>0$ independent of $T$ and a subsequence $\left(n^{\prime}\right)$ such that $\lim _{n^{\prime} \rightarrow \infty}\left\|\iota T R_{n^{\prime}}\right\|_{X, \ell_{\infty}\left(2^{-m j} F_{m}\right)} \leq C \beta_{j}$. 
We shall also need later the following consequence of [7, Lemma 3.1].

Lemma 3.5. Let $A, B, Z$ be quasi-Banach spaces and let $D$ be a dense subspace of $A$. Assume that $T \in \mathcal{L}(A, B)$ and let $\left(S_{n}\right) \subseteq \mathcal{L}(B, Z)$ such that $\sup \left\{\left\|S_{n}\right\|_{B, Z}: n \in \mathbb{N}\right\}<\infty$ and $\lim _{n \rightarrow \infty}\left\|S_{n} T u\right\|_{Z}=0$ for all $u \in D$. Let $\beta=\beta(T: A \rightarrow B)$. Then the following holds.

a) If $\beta=0$ then $\lim _{n \rightarrow \infty}\left\|S_{n} T\right\|_{A, Z}=0$.

b) If $\beta>0$ then there is a constant $C>0$ independent of $T$ and there is $L \in \mathbb{N}$ such that $\left\|S_{n} T\right\|_{A, Z} \leq C \beta$ for all $n \geq L$.

To complete the preparation for the central result of the paper, note that if $\Gamma$ is a $p$-Banach sequence lattice then it induces a $p$-norm $\|\cdot\|_{\tilde{\Gamma}}$ in $\mathbb{R}^{2 n+1}, n \in \mathbb{N}$. Indeed, if $x=\left(x_{k}\right)_{k=-n}^{n} \in \mathbb{R}^{2 n+1}$, put $\|x\|_{\tilde{\Gamma}}=\|\tilde{x}\|_{\Gamma}$ where $\tilde{x}=\sum_{k=-n}^{n} x_{k} e_{k}$ and $e_{k}=\left(\delta_{m}^{k}\right)$ with $\delta_{m}^{k}$ being the Kronecker delta. The unit ball $U=U_{\left(\mathbb{R}^{2 n+1},\|\cdot\|_{\tilde{\Gamma}}\right)}$ is compact in $\left(\mathbb{R}^{2 n+1},\|\cdot\|_{\tilde{\Gamma}}\right)$ and therefore for any $\varepsilon>0$ we can find an $\varepsilon$-net for $U$.

Subsequently, in order to make the arguments more transparent, constants are denoted by $c_{1}, c_{2}, \ldots$ In this way we emphasize the new constants that show up and a change in an existing constant.

Theorem 3.6. Let $\bar{A}=\left(A_{0}, A_{1}\right), \bar{B}=\left(B_{0}, B_{1}\right)$ be mutually closed quasi-Banach couples and let $T \in \mathcal{L}(\bar{A}, \bar{B})$. Let $0<q \leq \infty$ and $\mathbb{A}=\left(\alpha_{0}, \alpha_{\infty}\right) \in \mathbb{R}^{2}$ satisfying that

$$
\begin{cases}\alpha_{0}+1 / q<0 \leq \alpha_{\infty}+1 / q & \text { if } 0<q<\infty \\ \alpha_{0} \leq 0<\alpha_{\infty} & \text { if } q=\infty .\end{cases}
$$

Then we have:

a) $\beta\left(T:\left(A_{0}, A_{1}\right)_{1, q, \mathbb{A}} \rightarrow\left(B_{0}, B_{1}\right)_{1, q, \mathbb{A}}\right)=0$ if $\beta\left(T: A_{1} \rightarrow B_{1}\right)=0$,

b) $\beta\left(T:\left(A_{0}, A_{1}\right)_{1, q, \mathbb{A}} \rightarrow\left(B_{0}, B_{1}\right)_{1, q, \mathbb{A}}\right) \leq C \beta\left(T: A_{1} \rightarrow B_{1}\right)$ if $\beta\left(T: A_{0} \rightarrow B_{0}\right)=0$,

c) $\beta\left(T:\left(A_{0}, A_{1}\right)_{1, q, \mathbb{A}} \rightarrow\left(B_{0}, B_{1}\right)_{1, q, \mathbb{A}}\right) \leq C \beta\left(T: A_{1} \rightarrow B_{1}\right)\left(1+\left(\log \frac{\beta\left(T: A_{0} \rightarrow B_{0}\right)}{\beta\left(T: A_{1} \rightarrow B_{1}\right)}\right)^{+}\right)^{\alpha_{\infty}^{+}-\alpha_{0}}$ if $\beta\left(T: A_{j} \rightarrow B_{j}\right)>0$ for $j=0,1$.

Here $C>0$ is a constant independent of $\bar{A}, \bar{B}$ and $T$.

Proof. Without loss of generality we can suppose that the spaces $A_{0}, A_{1}, B_{0}, B_{1}$ are $p$-Banach for some $0<p \leq 1$. Furthermore, since $\bar{A}$ is mutually closed, by Theorem 2.1 , we have that

$$
\left(A_{0}, A_{1}\right)_{1, q, \mathbb{A}}=\left(A_{0}, A_{1}\right)_{\Lambda ; J} \text { with } \Lambda=\left(\ell_{p}, \ell_{p}\left(2^{-m}\right)\right)_{1, q, \mathbb{A}} .
$$

For each $m \in \mathbb{Z}$ let

$$
G_{m}=\left(A_{0} \cap A_{1}, J\left(2^{m}, \cdot ; A_{0}, A_{1}\right)\right), F_{m}=\left(B_{0}+B_{1}, K\left(2^{m}, \cdot ; B_{0}, B_{1}\right)\right) .
$$

The operator $\pi\left(u_{m}\right)=\sum_{m=-\infty}^{\infty} u_{m}$ (convergence in $A_{0}+A_{1}$ ) is surjective from $\Lambda\left(G_{m}\right)$ into $\left(A_{0}, A_{1}\right)_{\Lambda ; J}$ and it induces the quasi-norm of $\left(A_{0}, A_{1}\right)_{\Lambda ; J}$. On the other hand, as we pointed out before Lemma 3.4, if $\Delta=\ell_{q}\left(2^{-m} \ell^{\mathbb{A}}\left(2^{m}\right)\right)$ and $\iota b=(\ldots, b, b, b, b, \ldots)$, then $\iota$ is a metric injection from $\left(B_{0}, B_{1}\right)_{1, q, \mathbb{A}}$ into $\Delta\left(F_{m}\right)$. Let $\hat{T}=\iota T \pi: \Lambda\left(G_{m}\right) \rightarrow \Delta\left(F_{m}\right)$. According to (3.2) and (3.3), we get

$$
\beta\left(T: \bar{A}_{1, q, \mathbb{A}} \rightarrow \bar{B}_{1, q, \mathbb{A}}\right) \leq c_{1} \beta\left(\iota T: \bar{A}_{1, q, \mathbb{A}} \rightarrow \Delta\left(F_{m}\right)\right) \leq c_{2} \beta\left(\hat{T}: \Lambda\left(G_{m}\right) \rightarrow \Delta\left(F_{m}\right)\right) .
$$


With the aim of estimating the last measure of non-compactness, consider the couples

$$
\overline{\ell_{p}(G)}=\left(\ell_{p}\left(G_{m}\right), \ell_{p}\left(2^{-m} G_{m}\right)\right) \quad \text { and } \quad \overline{\ell_{\infty}(F)}=\left(\ell_{\infty}\left(F_{m}\right), \ell_{\infty}\left(2^{-m} F_{m}\right)\right)
$$

and note that $\pi \in \mathcal{L}\left(\ell_{p}\left(2^{-m j} G_{m}\right), A_{j}\right), j=0,1$, with quasi-norm less than or equal to 1 . On the other hand, $\iota \in \mathcal{L}\left(B_{j}, \ell_{\infty}\left(2^{-m j} F_{m}\right)\right), j=0,1$, also with quasi-norm less than or equal to 1. The relevant picture to keep in mind is

$$
\begin{array}{ccccccc}
\ell_{p}\left(G_{m}\right) & \stackrel{\pi}{\longrightarrow} & A_{0} & \stackrel{T}{\longrightarrow} & B_{0} & \stackrel{\iota}{\longrightarrow} & \ell_{\infty}\left(F_{m}\right) \\
\ell_{p}\left(2^{-m} G_{m}\right) & \stackrel{\pi}{\longrightarrow} & A_{1} & \stackrel{T}{\longrightarrow} & B_{1} & \stackrel{\iota}{\longrightarrow} & \ell_{\infty}\left(2^{-m} F_{m}\right) \\
\hline \hline \bar{\ell}_{p}(G)_{1, q, \mathbb{A}} & \stackrel{\pi}{\longrightarrow} & \bar{A}_{1, q, \mathbb{A}} & \stackrel{T}{\longrightarrow} & \bar{B}_{1, q, \mathbb{A}} & \stackrel{\iota}{\longrightarrow} & \bar{\ell}_{\infty}(F)_{1, q, \mathbb{A}}
\end{array}
$$

Moreover, by Lemmata 3.2 and 3.3, we have

$$
\Lambda\left(G_{m}\right) \hookrightarrow{\overline{\ell_{p}(G)}}_{1, q, \mathbb{A}} \quad \text { and } \quad{\overline{\ell_{\infty}(F)}}_{1, q, \mathbb{A}} \hookrightarrow \Delta\left(F_{m}\right) .
$$

On $\overline{\ell_{p}(G)}$ we can consider the following projections. For $n \in \mathbb{N}$ and $u=\left(u_{m}\right)$, let

$$
\begin{aligned}
P_{n} u & =\left(\ldots, 0,0, u_{-n}, u_{-n+1}, \ldots, u_{n-1}, u_{n}, 0,0, \ldots\right), \\
P_{n}^{+} u & =\left(\ldots, 0,0, u_{n+1}, u_{n+2}, u_{n+3}, \ldots\right), \\
P_{n}^{-} u & =\left(\ldots, u_{-n-3}, u_{-n-2}, u_{-n-1}, 0,0, \ldots\right) .
\end{aligned}
$$

These mappings have quasi-norm 1 when acting from $\ell_{p}\left(2^{-j m} G_{m}\right)$ into $\ell_{p}\left(2^{-j m} G_{m}\right), j=0,1$, or from $\Lambda\left(G_{m}\right)$ into $\Lambda\left(G_{m}\right)$. The identity operator $I$ on $\ell_{p}\left(G_{m}\right)+\ell_{p}\left(2^{-m} G_{m}\right)$ can be split as $I=P_{n}+P_{n}^{+}+P_{n}^{-}, n \in \mathbb{N}$. Moreover

$$
\left\|P_{n}^{+}\right\|_{\ell_{p}\left(G_{m}\right), \ell_{p}\left(2^{-m} G_{m}\right)}=2^{-(n+1)}=\left\|P_{n}^{-}\right\|_{\ell_{p}\left(2^{-m} G_{m}\right), \ell_{p}\left(G_{m}\right)} .
$$

On the couple $\overline{\ell_{\infty}(F)}$ we can consider similar sequences of projections with analogous properties. We denote them by $\left(Q_{n}\right),\left(Q_{n}^{+}\right),\left(Q_{n}^{-}\right)$.

Since

$$
\hat{T}=\hat{T} P_{n}+Q_{n} \hat{T}\left(P_{n}^{+}+P_{n}^{-}\right)+Q_{n}^{+} \hat{T} P_{n}^{-}+Q_{n}^{-} \hat{T} P_{n}^{+}+Q_{n}^{-} \hat{T} P_{n}^{-}+Q_{n}^{+} \hat{T} P_{n}^{+},
$$

we proceed to estimate the measure of non-compactness of each one of these six operators acting from $\Lambda\left(G_{m}\right)$ into $\Delta\left(F_{m}\right)$. Take any $\sigma_{j}>\beta\left(T: A_{j} \rightarrow B_{j}\right)$ and let $N \in \mathbb{N} \cup\{0\}$ such that $2^{N} \leq \sigma_{0} / \sigma_{1}<2^{N+1}$ if $\sigma_{1} \leq \sigma_{0}$ and $N=0$ if $\sigma_{0}<\sigma_{1}$.

For $\hat{T} P_{n}$ we have

$$
\beta\left(\hat{T} P_{n}: \Lambda\left(G_{m}\right) \rightarrow \Delta\left(F_{m}\right)\right) \leq c \beta\left(T \pi P_{n}: \Lambda\left(G_{m}\right) \rightarrow\left(B_{0}, B_{1}\right)_{\Lambda ; J}\right) .
$$

Let $\eta=\left\|\sum_{k=-n}^{n} \frac{e_{k}}{\left\|e_{k}\right\|_{\Lambda}}\right\|_{\Lambda}^{-1}$ and consider the quasi-norm $\|\cdot\|_{\tilde{\Lambda}}$ in $\mathbb{R}^{2 n+1}$. We can find $\Upsilon=$ $\left\{\lambda^{1}, \ldots, \lambda^{s}\right\} \subseteq U_{\left(\mathbb{R}^{2 n+1},\|\cdot\|_{\tilde{\Lambda}}\right)}$ such that

$$
U_{\left(\mathbb{R}^{2 n+1},\|\cdot\|_{\tilde{\Lambda}}\right)} \subseteq \bigcup_{d=1}^{s}\left\{\lambda^{d}+\eta U_{\left(\mathbb{R}^{2 n+1},\|\cdot\|_{\tilde{\Lambda}}\right)}\right\} .
$$

For each $\lambda^{d}=\left(\lambda_{k}^{d}\right)_{k=-n}^{n}$, let

$$
\varphi_{k}^{j}=\varphi_{k, \lambda^{d}}^{j}=\left(\frac{\eta}{\left\|e_{k}\right\|_{\Lambda}}+\left|\lambda_{k}^{d}\right|\right) 2^{-k j}, j=0,1 .
$$


Besides, there are finite sets $\Sigma_{j}=\left\{h_{1}^{j}, \ldots, h_{L_{j}}^{j}\right\} \subseteq B_{j}$ such that

$$
T\left(U_{A_{j}}\right) \subseteq \bigcup_{\ell=1}^{L_{j}}\left\{h_{\ell}^{j}+\sigma_{j} U_{B_{j}}\right\}, j=0,1 .
$$

Given any $\lambda^{d} \in \Upsilon, h_{\ell}^{0} \in \Sigma_{0}$ and $h_{y}^{1} \in \Sigma_{1}$, for any $-n \leq k \leq n$, let $g_{k}$ be an element of $\left(\varphi_{k}^{0} h_{\ell}^{0}+\varphi_{k}^{0} \sigma_{0} U_{B_{0}}\right) \cap\left(\varphi_{k}^{1} h_{y}^{1}+\varphi_{k}^{1} \sigma_{1} U_{B_{1}}\right)$ provided the intersection is non-empty and let $g_{k}=0$ otherwise. Denote by $\Phi$ the collection of all sums $\sum_{k=-n}^{n} g_{k}$ of the elements $g_{k}$. Note that $\Phi$ is a finite set. Now we show that $\Phi$ is a suitable net for $T \pi P_{n}$.

Given any $u=\left(u_{m}\right) \in U_{\Lambda\left(G_{m}\right)}$, there is $\lambda^{d} \in \Upsilon$ such that

$$
\left|J\left(2^{k}, u_{k}\right)-\lambda_{k}^{d}\right|\left\|e_{k}\right\|_{\Lambda} \leq\left\|\left(J\left(2^{k}, u_{k}\right)-\lambda_{k}^{d}\right)\right\|_{\tilde{\Lambda}} \leq \eta,-n \leq k \leq n .
$$

Hence, $\left|J\left(2^{k}, u_{k}\right)\right| \leq \frac{\eta}{\left\|e_{k}\right\|_{\Lambda}}+\left|\lambda_{k}^{d}\right|=\varphi_{k}^{0}$ and so $\left\|u_{k}\right\|_{A_{j}} \leq \varphi_{k}^{j}, j=0,1,-n \leq k \leq n$. We can find $h_{\ell}^{0} \in \Sigma_{0}$ and $h_{y}^{1} \in \Sigma_{1}$ such that

$$
\left\|T u_{k}-\varphi_{k}^{0} h_{\ell}^{0}\right\|_{B_{0}} \leq \varphi_{k}^{0} \sigma_{0},\left\|T u_{k}-\varphi_{k}^{1} h_{y}^{1}\right\|_{B_{1}} \leq \varphi_{k}^{1} \sigma_{1} .
$$

Thus,

$$
\left(\varphi_{k}^{0} h_{\ell}^{0}+\varphi_{k}^{0} \sigma_{0} U_{B_{0}}\right) \cap\left(\varphi_{k}^{1} h_{y}^{1}+\varphi_{k}^{1} \sigma_{1} U_{B_{1}}\right) \neq \emptyset
$$

and for the corresponding $g_{k}$ we obtain that

$$
\begin{aligned}
J\left(2^{k+N}, T u_{k}-g_{k}\right) \leq & \max \left\{\left\|T u_{k}-\varphi_{k}^{0} h_{\ell}^{0}\right\|_{B_{0}}^{p}+\left\|\varphi_{k}^{0} h_{\ell}^{0}-g_{k}\right\|_{B_{0}}^{p},\right. \\
& \left.2^{(k+N) p}\left(\left\|T u_{k}-\varphi_{k}^{1} h_{y}^{1}\right\|_{B_{1}}^{p}+\left\|\varphi_{k}^{1} h_{y}^{1}-g_{k}\right\|_{B_{1}}^{p}\right)\right\}^{1 / p} \\
& \leq 2^{1 / p-1} \max \left\{\sigma_{0}, 2^{N} \sigma_{1}\right\} \varphi_{k}^{0} .
\end{aligned}
$$

For $g=\sum_{k=-n}^{n} g_{k} \in \Phi$, it follows that

$$
\begin{aligned}
\left\|T \pi P_{n} u-g\right\|_{\left(B_{0}, B_{1}\right)_{\Lambda ; J}} & =\left\|\sum_{k=-n}^{n}\left(T u_{k}-g_{k}\right)\right\|_{\left(B_{0}, B_{1}\right)_{\Lambda ; J}} \\
& \leq\left\|\tau_{-N}\left(\ldots, 0,0, J\left(2^{-n+N}, T u_{-n}-g_{-n}\right), \ldots, J\left(2^{n+N}, T u_{n}-g_{n}\right), 0,0, \ldots\right)\right\|_{\Lambda} \\
& \leq 2^{1 / p-1}\left\|\tau_{-N}\right\|_{\Lambda, \Lambda} \max \left\{\sigma_{0}, 2^{N} \sigma_{1}\right\}\left\|\left(\ldots, 0,0, \varphi_{-n}^{0}, \ldots, \varphi_{n}^{0}, 0,0, \ldots\right)\right\|_{\Lambda} \\
& \leq c_{3}\left\|\tau_{-N}\right\|_{\Lambda, \Lambda} \max \left\{\sigma_{0}, 2^{N} \sigma_{1}\right\} .
\end{aligned}
$$

By our choice of $N$ and Lemma 2.3 we conclude that

$$
\beta\left(\hat{T} P_{n}: \Lambda\left(G_{m}\right) \rightarrow \Delta\left(F_{m}\right)\right) \leq 2^{1 / p} c_{3} \sigma_{1}\left(1+\left(\log \frac{\sigma_{0}}{\sigma_{1}}\right)^{+}\right)^{\alpha_{\infty}^{+}-\alpha_{0}} .
$$

As for $Q_{n} \hat{T}\left(P_{n}^{+}+P_{n}^{-}\right)$we get

$$
\beta\left(Q_{n} \hat{T}\left(P_{n}^{+}+P_{n}^{-}\right): \Lambda\left(G_{m}\right) \rightarrow \Delta\left(F_{m}\right)\right) \leq c_{4} \beta\left(Q_{n} \iota T: \bar{A}_{1, q, \mathbb{A}} \rightarrow \Delta\left(F_{m}\right)\right) .
$$

To estimate the last measure of non-compactness let $\sigma_{0}, \sigma_{1}$ and $N$ be as before. Put $\eta=$ $\left\|\sum_{k=-n}^{n} \frac{e_{k}}{\left\|e_{k}\right\|_{\Delta}}\right\|_{\Delta}^{-1}$ and choose $\Psi=\left\{\mu^{1}, \ldots, \mu^{s}\right\} \subseteq U_{\left(\mathbb{R}^{2 n+1},\|\cdot\|_{\tilde{\Delta}}\right)}$ such that for any $x \in U_{\left(\mathbb{R}^{2 n+1},\|\cdot\|_{\tilde{\Delta}}\right)}$ there is $\mu^{d}$ such that $\left\|x-\mu^{d}\right\|_{\tilde{\Delta}}<\eta$. Let associate to each $\mu^{d}=\left(\mu_{k}^{d}\right)_{k=-n}^{n}$ the numbers

$$
\psi_{k}^{j}=\psi_{k, \mu^{d}}^{j}=\left\|\tau_{-N}\right\|_{\Delta, \Delta}\left(\frac{\eta}{\left\|e_{k}\right\|_{\Delta}}+\left|\mu_{k}^{d}\right|\right) 2^{-(k-N) j}, j=0,1
$$


Choose a $\sigma_{j}$-net $\Sigma_{j}=\left\{h_{1}^{j}, \ldots, h_{L_{j}}^{j}\right\}$ for $T\left(U_{A_{j}}\right)$ in $B_{j}, j=0,1$, and given any $\mu^{d} \in \Psi, h_{\ell}^{0} \in \Sigma_{0}$ and $h_{y}^{1} \in \Sigma_{1}$, let $z^{d, \ell, y}=\left(z_{m}^{d, \ell, y}\right)_{m \in \mathbb{Z}}$ the sequence of vectors defined by

$$
z_{m}^{d, \ell, y}= \begin{cases}0 & \text { if } m \notin[-n, n], \\ \psi_{m}^{0} h_{\ell}^{0}+\psi_{m}^{1} h_{y}^{1} & \text { if }-n \leq m \leq n .\end{cases}
$$

Clearly, the collection of these $z^{d, \ell, y}$ forms a finite subset $\Omega$ of $\Delta\left(F_{m}\right)$. We are going to show that $\Omega$ is a suitable net for $Q_{n} \iota T$.

Take any $a \in U_{\bar{A}_{1, q, \mathbb{A}}}$. Since

$$
\left\|\left(K\left(2^{m-N}, a\right)\right)\right\|_{\Delta} \leq\left\|\tau_{-N}\right\|_{\Delta, \Delta}\|a\|_{\bar{A}_{1, q, \mathbb{A}}} \leq\left\|\tau_{-N}\right\|_{\Delta, \Delta},
$$

there is $\mu^{d} \in \Psi$ such that

$$
\left\|\left(K\left(2^{m-N}, a\right)-\left\|\tau_{-N}\right\|_{\Delta, \Delta} \mu_{m}^{d}\right)_{m=-n}^{n}\right\|_{\tilde{\Delta}}<\eta\left\|\tau_{-N}\right\|_{\Delta, \Delta}
$$

So,

$$
\left|K\left(2^{m-N}, a\right)-\left\|\tau_{-N}\right\|_{\Delta, \Delta} \mu_{m}^{d}\right|\left\|e_{m}\right\|_{\Delta}<\eta\left\|\tau_{-N}\right\|_{\Delta, \Delta}, \quad-n \leq m \leq n .
$$

This yields that $K\left(2^{m-N}, a\right)<\psi_{m}^{0},-n \leq m \leq n$. We can decompose $a=a_{0, m}+a_{1, m}$ with $a_{j, m} \in A_{j}, j=0,1$, and

$$
\left\|a_{0, m}\right\|_{A_{0}}+2^{m-N}\left\|a_{1, m}\right\|_{A_{1}}<\psi_{m}^{0} .
$$

It follows that for some $h_{\ell}^{0} \in \Sigma_{0}, h_{y}^{1} \in \Sigma_{1}$

$$
\left\|T a_{0, m}-\psi_{m}^{0} h_{\ell}^{0}\right\|_{B_{0}} \leq \psi_{m}^{0} \sigma_{0} \text { and }\left\|T a_{1, m}-\psi_{m}^{1} h_{y}^{1}\right\|_{B_{1}} \leq \psi_{m}^{1} \sigma_{1},-n \leq m \leq n .
$$

Let $z=z^{d, \ell, y}$ be the element of $\Omega$ associated to $\mu^{d}, h_{\ell}^{0}$ and $h_{y}^{1}$. Then,

$$
\begin{aligned}
\left\|Q_{n} \iota T a-z\right\|_{\Delta\left(F_{m}\right)} & =\left\|\left(K\left(2^{m}, T a-z_{m}\right)\right)_{m=-n}^{n}\right\|_{\tilde{\Delta}} \\
& \leq\left\|\left(\left\|T a_{0, m}-\psi_{m}^{0} h_{\ell}^{0}\right\|_{B_{0}}+2^{m}\left\|T a_{1, m}-\psi_{m}^{1} h_{y}^{1}\right\|_{B_{1}}\right)_{m=-n}^{n}\right\|_{\tilde{\Delta}} \\
& \leq\left\|\left(\psi_{m}^{0} \sigma_{0}+2^{m} \psi_{m}^{1} \sigma_{1}\right)_{m=-n}^{n}\right\|_{\tilde{\Delta}} \leq 2 c_{\Delta}\left\|\tau_{-N}\right\|_{\Delta, \Delta}\left(\sigma_{0}+2^{N} \sigma_{1}\right) .
\end{aligned}
$$

By the choice of $N$ and Lemma 2.2, we derive that

$$
\beta\left(Q_{n} \iota T: \bar{A}_{1, q, \mathbb{A}} \rightarrow \Delta\left(F_{m}\right)\right) \leq 8 c_{\Delta} \sigma_{1}\left(1+\left(\log \frac{\sigma_{0}}{\sigma_{1}}\right)^{+}\right)^{\alpha_{\infty}^{+}-\alpha_{0}}
$$

As for $Q_{n}^{+} \hat{T} P_{n}^{-}$we are going to estimate the measure of non-compactness by the quasi-norm of the operator and then to show that it tends to 0 as $n \rightarrow \infty$. We have $\left\|Q_{n}^{+} \hat{T} P_{n}^{-}\right\|_{\ell_{p}\left(G_{m}\right), \ell_{\infty}\left(F_{m}\right)} \leq$ $\|T\|_{A_{0}, B_{0}}$. On the other hand, the factorization

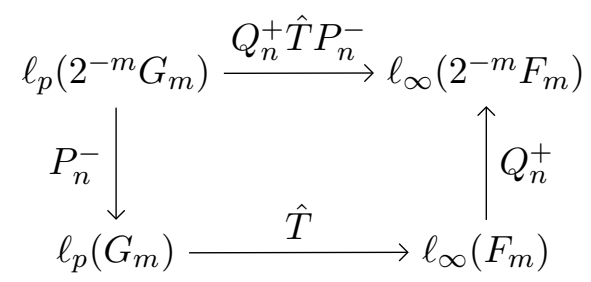


and (3.5) give that

$$
\left\|Q_{n}^{+} \hat{T} P_{n}^{-}\right\|_{\ell_{p}\left(2^{-m} G_{m}\right), \ell_{\infty}\left(2^{-m} G_{m}\right)} \leq 2^{-(n+1)}\|\hat{T}\|_{\ell_{p}\left(G_{m}\right), \ell_{\infty}\left(F_{m}\right)} 2^{-(n+1)} \leq 2^{-2(n+1)}\|T\|_{A_{0}, B_{0}} .
$$

Hence, according to Lemmata 3.2, 3.3 and estimate (3.1), we get

$$
\begin{aligned}
\beta\left(Q_{n}^{+} \hat{T} P_{n}^{-}: \Lambda\left(G_{m}\right) \rightarrow \Delta\left(F_{m}\right)\right) & \leq c\left\|Q_{n}^{+} \hat{T} P_{n}^{-}\right\|_{{\overline{\ell_{p}}(G)_{1, q, \mathbb{A}}, \bar{\ell}_{\infty}(F)_{1, q, \mathbb{A}}}} \\
& \leq c 2^{-2(n+1)}\|T\|_{A_{0}, B_{0}}\left(1+\log 2^{2(n+1)}\right)^{\alpha_{\infty}^{+}-\alpha_{0}} \rightarrow 0 \text { as } n \rightarrow \infty
\end{aligned}
$$

As for $Q_{n}^{-} \hat{T} P_{n}^{+}$, we observe that

$$
\left\|P_{n}^{+}\right\|_{\ell_{p}\left(G_{m}\right), \ell_{p}\left(2^{-m} G_{m}\right)}=2^{-(n+1)} \text { and }\left\|P_{n}^{+}\right\|_{\ell_{p}\left(2^{-m} G_{m}\right), \ell_{p}\left(2^{-m} G_{m}\right)}=1,
$$

hence $\left\|P_{n}^{+}\right\|_{\Lambda\left(G_{m}\right), \ell_{p}\left(2^{-m} G_{m}\right)} \leq c_{5}$. Similarly, $\left\|Q_{n}^{-}\right\|_{\ell_{\infty}\left(2^{-m} F_{m}\right), \Delta\left(F_{m}\right)} \leq c_{6}$. Using the diagram

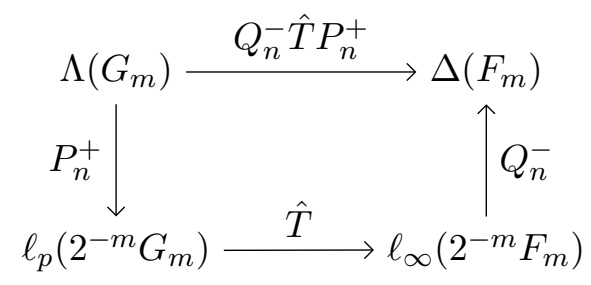

we derive that

$$
\begin{aligned}
\beta\left(Q_{n}^{-} \hat{T} P_{n}^{+}: \Lambda\left(G_{m}\right) \rightarrow \Delta\left(F_{m}\right)\right) & \leq c_{5} c_{6} \beta\left(\hat{T}: \ell_{p}\left(2^{-m} G_{m}\right) \rightarrow \ell_{\infty}\left(2^{-m} F_{m}\right)\right) \\
& \leq c_{5} c_{6} \sigma_{1} \leq c_{5} c_{6} \sigma_{1}\left(1+\left(\log \frac{\sigma_{0}}{\sigma_{1}}\right)^{+}\right)^{\alpha_{\infty}^{+}-\alpha_{0}} .
\end{aligned}
$$

The remaining two operators can be estimated with the help of Lemmata 3.4 and 3.5. Using the factorization

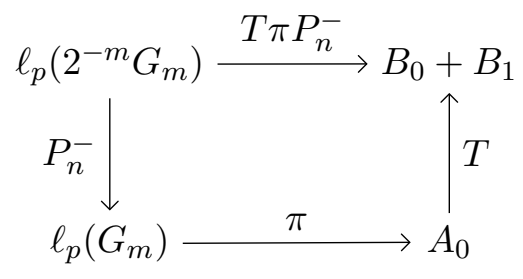

we get

$$
\left\|T \pi P_{n}^{-}\right\|_{\ell_{p}\left(2^{-m} G_{m}\right), B_{0}+B_{1}} \leq 2^{-(n+1)}\|T\|_{A_{0}, B_{0}+B_{1}} \rightarrow 0 \text { as } n \rightarrow \infty .
$$

Whence, Lemma 3.4 yields that there is $c_{7}>0$ and a subsequence $\left(n^{\prime}\right)$ such that

$$
\lim _{n^{\prime} \rightarrow \infty}\left\|\hat{T} P_{n^{\prime}}^{-}\right\|_{\ell_{p}\left(2^{-m} G_{m}\right), \ell_{\infty}\left(2^{-m} F_{m}\right)} \leq c_{7} \beta\left(T \pi: \ell_{p}\left(2^{-m} G_{m}\right) \rightarrow B_{1}\right) \leq c_{7} \sigma_{1} .
$$

On the other hand, let $D$ be a subset of $\ell_{p}\left(G_{m}\right)$ formed by all sequences having only a finite number of coordinates different from 0 . Clearly, $D$ is dense in $\ell_{p}\left(G_{m}\right)$ and if $u \in D$ then

$$
\left\|Q_{n}^{-} \hat{T} u\right\|_{\ell_{\infty}\left(F_{m}\right)} \leq 2^{-(n+1)}\|\hat{T} u\|_{\ell_{\infty}\left(2^{-m} F_{m}\right)} \rightarrow 0 \text { as } n \rightarrow \infty .
$$

According to Lemma 3.5, there is $c_{8}>0$ and $L \in \mathbb{N}$ such that for all $n \geq L$

$$
\left\|Q_{n}^{-} \hat{T}\right\|_{\ell_{p}\left(G_{m}\right), \ell_{\infty}\left(F_{m}\right)} \leq c_{8} \beta\left(\hat{T}: \ell_{p}\left(G_{m}\right) \rightarrow \ell_{\infty}\left(F_{m}\right)\right) \leq c_{8} \sigma_{0} .
$$


Using now Lemmata 3.2, 3.3 and (3.1), if $n^{\prime}>L$ we derive that

$$
\beta\left(Q_{n^{\prime}}^{-} \hat{T} P_{n^{\prime}}^{-}: \Lambda\left(G_{m}\right) \rightarrow \Delta\left(F_{m}\right)\right) \leq c_{9}\left\|Q_{n^{\prime}}^{-} \hat{T} P_{n^{\prime}}^{-}\right\|_{{\overline{\ell_{p}}(G)}_{1, q, \mathbb{A}}, \bar{\ell}_{\infty}(F)_{1, q, \mathbb{A}}} \leq c \sigma_{1}\left(1+\left(\log \frac{\sigma_{0}}{\sigma_{1}}\right)^{+}\right)^{\alpha_{\infty}^{+}-\alpha_{0}} .
$$

With a similar argument one can show that there is another subsequence that we also denote by $\left(n^{\prime}\right)$ and another positive integer that we also call $L$ such that if $n^{\prime}>L$ we have that

$$
\beta\left(Q_{n^{\prime}}^{+} \hat{T} P_{n^{\prime}}^{+}: \Lambda\left(G_{m}\right) \rightarrow \Delta\left(F_{m}\right)\right) \leq c \sigma_{1}\left(1+\left(\log \frac{\sigma_{0}}{\sigma_{1}}\right)^{+}\right)^{\alpha_{\infty}^{+}-\alpha_{0}}
$$

Collecting all these estimates we derive that there is a constant $C>0$ independent of $\bar{A}, \bar{B}$ and $T$ such that if we split the operator as in (3.6) with a suitable $n$ then

$$
\beta\left(\hat{T}: \Lambda\left(G_{m}\right) \rightarrow \Delta\left(F_{m}\right)\right) \leq C \sigma_{1}\left(1+\left(\log \frac{\sigma_{0}}{\sigma_{1}}\right)^{+}\right)^{\alpha_{\infty}^{+}-\alpha_{0}} .
$$

Now take $\sigma_{j}=(1+\varepsilon) \beta\left(T: A_{j} \rightarrow B_{j}\right)$ if $\beta\left(T: A_{j} \rightarrow B_{j}\right)>0$ and $\sigma_{j}=\varepsilon$ otherwise. Letting $\varepsilon \rightarrow 0$ and using (3.4) the result follows.

Next we get rid of the assumption on the couples of being mutually closed.

Theorem 3.7. Let $\bar{A}=\left(A_{0}, A_{1}\right), \bar{B}=\left(B_{0}, B_{1}\right)$ be quasi-Banach couples and let $T \in \mathcal{L}(\bar{A}, \bar{B})$. If $q$ and $\mathbb{A}$ are as in Theorem 3.6, then the conclusion of that theorem is still valid.

Proof. The quasi-Banach couples $\overline{A^{\sim}}=\left(A_{0}^{\sim}, A_{1}^{\sim}\right), \overline{B^{\sim}}=\left(B_{0}^{\sim}, B_{1}^{\sim}\right)$ are mutually closed and, by (2.5), $\bar{A}_{1, q, \mathbb{A}}={\overline{A^{\sim}}}_{1, q, \mathbb{A}}$ and $\bar{B}_{1, q, \mathbb{A}}={\overline{B^{\sim}}}_{1, q, \mathbb{A}}$. Furthermore, $T$ belongs to $\mathcal{L}\left(\overline{A^{\sim}}, \overline{B^{\sim}}\right)$ and, according to Lemma 3.1 ,

$$
\beta\left(T: A_{j}^{\sim} \rightarrow B_{j}^{\sim}\right) \leq \max \left\{c_{B_{0}}, c_{B_{1}}\right\} \beta\left(T: A_{j} \rightarrow B_{j}\right), j=0,1 .
$$

Consequently, Theorem 3.6 yields the result.

As a direct consequence of (2.1) and Theorem 3.7 we get the following result for logarithmic methods with $\theta=0$.

Theorem 3.8. Let $\bar{A}=\left(A_{0}, A_{1}\right), \bar{B}=\left(B_{0}, B_{1}\right)$ be quasi-Banach couples and let $T \in \mathcal{L}(\bar{A}, \bar{B})$. Let $0<q \leq \infty$ and $\mathbb{A}=\left(\alpha_{0}, \alpha_{\infty}\right) \in \mathbb{R}^{2}$ satisfying that

$$
\begin{cases}\alpha_{\infty}+1 / q<0 \leq \alpha_{0}+1 / q & \text { if } 0<q<\infty \\ \alpha_{\infty} \leq 0<\alpha_{0} & \text { if } q=\infty .\end{cases}
$$

Then we have:

a) $\beta\left(T:\left(A_{0}, A_{1}\right)_{0, q, \mathbb{A}} \rightarrow\left(B_{0}, B_{1}\right)_{0, q, \mathbb{A}}\right)=0$ if $\beta\left(T: A_{0} \rightarrow B_{0}\right)=0$,

b) $\beta\left(T:\left(A_{0}, A_{1}\right)_{0, q, \mathbb{A}} \rightarrow\left(B_{0}, B_{1}\right)_{0, q, \mathbb{A}}\right) \leq C \beta\left(T: A_{0} \rightarrow B_{0}\right)$ if $\beta\left(T: A_{1} \rightarrow B_{1}\right)=0$,

c) $\beta\left(T:\left(A_{0}, A_{1}\right)_{0, q, \mathbb{A}} \rightarrow\left(B_{0}, B_{1}\right)_{0, q, \mathbb{A}}\right) \leq C \beta\left(T: A_{0} \rightarrow B_{0}\right)\left(1+\left(\log \frac{\beta\left(T: A_{1} \rightarrow B_{1}\right)}{\beta\left(T: A_{0} \rightarrow B_{0}\right)}\right)^{+}\right)^{\alpha_{0}^{+}-\alpha_{\infty}}$ if $\beta\left(T: A_{j} \rightarrow B_{j}\right)>0$ for $j=0,1$.

Here $C>0$ is a constant independent of $\bar{A}, \bar{B}$ and $T$. 
Another consequence of Theorem 3.7 is the following compactness result. Its novelty is that in the source and the target we have quasi-Banach couples. Note that we require weaker assumptions on $\mathbb{A}$ than in Theorem 3.7.

Theorem 3.9. Let $\bar{A}=\left(A_{0}, A_{1}\right), \bar{B}=\left(B_{0}, B_{1}\right)$ be quasi-Banach couples. Assume that $0<q \leq$ $\infty$ and $\mathbb{A}=\left(\alpha_{0}, \alpha_{\infty}\right) \in \mathbb{R}^{2}$ satisfy that

$$
\begin{cases}\alpha_{0}+1 / q<0 & \text { if } 0<q<\infty \\ \alpha_{0} \leq 0 & \text { if } q=\infty\end{cases}
$$

If $T \in \mathcal{L}(\bar{A}, \bar{B})$ and $T: A_{1} \rightarrow B_{1}$ is compact, then $T:\left(A_{0}, A_{1}\right)_{1, q, \mathbb{A}} \rightarrow\left(B_{0}, B_{1}\right)_{1, q, \mathbb{A}}$ is also compact.

Proof. Step 1. If $0 \leq \alpha_{\infty}+1 / q$ and $0<q<\infty$, or $0<\alpha_{\infty}$ and $q=\infty$, then the result follows from Theorem 3.7.

Step 2. Suppose now that $\alpha_{\infty}+1 / q<0$ and $0<q<\infty$, or $\alpha_{\infty} \leq 0$ and $q=\infty$. Take any $\alpha>-1 / q$. The argument in [20, Corollary 2.5] for Banach couples still work in the quasi-Banach context showing that

$$
\left(A_{0}, A_{1}\right)_{1, q, \mathbb{A}}=\left(A_{0}+A_{1}, A_{1}\right)_{1, q,\left(\alpha_{0}, \alpha\right)} \text { and }\left(B_{0}, B_{1}\right)_{1, q, \mathbb{A}}=\left(B_{0}+B_{1}, B_{1}\right)_{1, q,\left(\alpha_{0}, \alpha\right)} .
$$

Since $T \in \mathcal{L}\left(\left(A_{0}+A_{1}, A_{1}\right),\left(B_{0}+B_{1}, B_{1}\right)\right)$, the result established in the Step 1 yields that

$$
T:\left(A_{0}, A_{1}\right)_{1, q, \mathbb{A}} \rightarrow\left(B_{0}, B_{1}\right)_{1, q, \mathbb{A}} \text { compactly. }
$$

The compactness theorem for $\theta=0$ reads as follows.

Theorem 3.10. Let $\bar{A}=\left(A_{0}, A_{1}\right), \bar{B}=\left(B_{0}, B_{1}\right)$ be quasi-Banach couples. Assume that $0<$ $q \leq \infty$ and $\mathbb{A}=\left(\alpha_{0}, \alpha_{\infty}\right) \in \mathbb{R}^{2}$ satisfy that

$$
\begin{cases}\alpha_{\infty}+1 / q<0 & \text { if } 0<q<\infty \\ \alpha_{\infty} \leq 0 & \text { if } q=\infty .\end{cases}
$$

If $T \in \mathcal{L}(\bar{A}, \bar{B})$ and $T: A_{0} \rightarrow B_{0}$ is compact, then $T:\left(A_{0}, A_{1}\right)_{0, q, \mathbb{A}} \rightarrow\left(B_{0}, B_{1}\right)_{0, q, \mathbb{A}}$ compactly.

Remark 3.11. The compactness result of Cwikel [23] and Cobos, Kühn and Schonbek [18] shows that if any restriction of the operator is compact, then the interpolated operator by the classical real method is also compact. However, this is not the case for logarithmic methods with $\theta=0$ or 1 . Theorem 3.10 does not hold if we move the compactness assumption from $T: A_{0} \rightarrow B_{0}$ to $T: A_{1} \rightarrow B_{1}$. A counterexample can be found in [13, Remark 2.4]. A similar observation applies to Theorem 3.9 and a counterexample can be found in [16, Counterexample 7.11].

Next we apply the abstract results to derive quantitative versions of results of Edmunds and Opic (see [28, Corollary 4 and Theorem 6]). We work with function spaces on $\sigma$-finite measure spaces and the range for parameters $q_{0}, q_{1}, q$ is broader than in [28].

Let $(R, \mu)$ be a $\sigma$-finite measure spaces. For $0<p, q \leq \infty$ and $\mathbb{A}=\left(\alpha_{0}, \alpha_{\infty}\right) \in \mathbb{R}^{2}$, the generalized Lorentz-Zygmund space $L_{p, q}(\log L)_{\mathbb{A}}(R)$ consists of all (classes of) measurable functions $f$ on $R$ having a finite quasi-norm

$$
\|f\|_{L_{p, q}(\log L)_{\mathbb{A}}(R)}=\left(\int_{0}^{\mu(R)}\left[t^{1 / p} \ell^{\mathbb{A}}(t) f^{*}(t)\right]^{q} \frac{d t}{t}\right)^{1 / q}
$$

where $f^{*}$ is the non-increasing rearrangement of $f$. See $[3,38,25]$. 
Corollary 3.12. Let $(R, \mu)$ and $(S, \nu)$ be $\sigma$-finite measure spaces. Take $1 \leq p_{0}<p_{1}<\infty$, $0<q_{0}<q_{1}<\infty, 0<q<\infty$ and $\mathbb{A}=\left(\alpha_{0}, \alpha_{\infty}\right) \in \mathbb{R}^{2}$ satisfying that $\alpha_{0}+1 / q<0<\alpha_{\infty}+1 / q$. Let $T \in \mathcal{L}\left(\left(L_{p_{0}}(R), L_{p_{1}}(R)\right),\left(L_{q_{0}}(S), L_{q_{1}}(S)\right)\right)$, put $\beta_{j}=\beta\left(T: L_{p_{j}}(R) \rightarrow L_{q_{j}}(S)\right), j=0,1$, and

$$
\beta=\beta\left(T: L_{p_{1}, q}(\log L)_{\mathbb{A}+\frac{1}{\min \left(p_{1}, q\right)}}(R) \rightarrow L_{q_{1}, q}(\log L)_{\mathbb{A}+\frac{1}{\max \left(q_{1}, q\right)}}(S)\right) .
$$

Then there is a constant $C>0$ independent of $T$ such that

a) $\beta \leq C \beta_{1}\left(1+\left(\log \frac{\beta_{0}}{\beta_{1}}\right)^{+}\right)^{\alpha_{\infty}^{+}-\alpha_{0}}$ if $\beta_{j}>0$ for $j=0,1$;

b) $\beta \leq C \beta_{1}$ if $\beta_{0}=0$;

c) $\beta=0$ if $\beta_{1}=0$.

Proof. Reiteration results of Evans and Opic [29, Theorems 4.7, 5.9 and Corollary 8.4] yield that

$$
L_{p_{1}, q}(\log L)_{\mathbb{A}+\frac{1}{\min \left(p_{1}, q\right)}}(R) \hookrightarrow\left(L_{p_{0}}(R), L_{p_{1}}(R)\right)_{1, q, \mathbb{A}}
$$

and

$$
\left(L_{q_{0}}(S), L_{q_{1}}(S)\right)_{1, q, \mathbb{A}} \hookrightarrow L_{q_{1}, q}(\log L)_{\mathbb{A}+\frac{1}{\max \left(q_{1}, q\right)}}(S) .
$$

Whence, the result follows from Theorem 3.7.

Corollary 3.13. Let $(R, \mu),(S, \nu)$ be $\sigma$-finite measure spaces. Take $1<p_{0}<p_{1} \leq \infty, 0<$ $q_{0}<q_{1}<\infty, 0<q<\infty$ and $\mathbb{A}=\left(\alpha_{0}, \alpha_{\infty}\right) \in \mathbb{R}^{2}$ satisfying that $\alpha_{\infty}+1 / q<0<\alpha_{0}+1 / q$. Let $T \in \mathcal{L}\left(\left(L_{p_{0}}(R), L_{p_{1}}(R)\right),\left(L_{q_{0}}(S), L_{q_{1}}(S)\right)\right)$, put $\beta_{j}=\beta\left(T: L_{p_{j}}(R) \rightarrow L_{q_{j}}(S)\right), j=0,1$, and

$$
\beta=\beta\left(T: L_{p_{0}, q}(\log L)_{\mathbb{A}+\frac{1}{\min \left(p_{0}, q\right)}}(R) \rightarrow L_{q_{0}, q}(\log L)_{\mathbb{A}+\frac{1}{\max \left(q_{0}, q\right)}}(S)\right) .
$$

Then there exists a constant $C>0$ independent of $T$ such that

a) $\beta \leq C \beta_{0}\left(1+\left(\log \frac{\beta_{1}}{\beta_{0}}\right)^{+}\right)^{\alpha_{0}^{+}-\alpha_{\infty}}$ if $\beta_{j}>0$, for $j=0,1$;

b) $\beta \leq C \beta_{0}$ if $\beta_{1}=0$;

c) $\beta=0$ if $\beta_{0}=0$.

Proof. We can proceed as in the previous corollary but using now Theorem 3.8 .

Funding.- This work was supported in part by Agencia Estatal de Investigación y Fondo Europeo de Desarrollo Regional [grant MTM2017-84508-P]; and the Spanish Ministerio de Educación, Cultura y Deporte [grant FPU16/02420 to B.F.B.].

Acknowledgments.- The authors would like to thank the referee for his/her useful remarks. 


\section{References}

[1] W. Amrein, A. Boutet de Monvel and V. Georgescu, $C_{0}$-Groups, Commutator Methods and Spectral Theory of N-Body Hamiltonians, Springer, Basel, 1996.

[2] C. Bennett and R. Sharpley, Interpolation of Operators, Academic Press, Boston, 1988.

[3] C. Bennett and K. Rudnick, On Lorentz-Zygmund spaces, Dissertationes Math. 175 (1980), 1-72.

[4] J. Bergh and J. Löfström, Interpolation Spaces. An introduction, Springer, Berlin, 1976.

[5] B.F. Besoy, On compactness theorems for logarithmic interpolation methods, Proceedings of the Conference Function Spaces XII, Banach Center Publ. (to appear).

[6] B.F. Besoy and F. Cobos, Duality for logarithmic interpolation spaces when $0<q<1$ and applications, J. Math. Anal. Appl. 466 (2018), 373-399.

[7] B.F. Besoy and F. Cobos, Interpolation of the measure of non-compactness of bilinear operators among quasi-Banach spaces, J. Approx. Theory 243 (2019), 25-44.

[8] Y. Brudnyĩ and N. Krugljak, Interpolation Functors and Interpolation Spaces, Vol. 1, North-Holland, Amsterdam, 1991.

[9] P.L. Butzer and H. Berens, Semi-Groups of Operators and Approximation, Springer-Verlag, Berlin, 1967.

[10] A.P. Calderón, Intermediate spaces and interpolation, the complex method, Studia Math. 24 (1964), $113-190$.

[11] F. Cobos, Interpolation theory and compactness, in Function Spaces, Inequalities and Interpolation. Edited by J. Lukes and L. Pick, Paseky, Prague 2009, pp. 31-75.

[12] F. Cobos, L.M. Fernández-Cabrera and A. Martínez, Abstract K and J spaces and measure of non-compactness, Math. Nachr. 280 (2007), 1698-1708.

[13] F. Cobos, L.M. Fernández-Cabrera and A. Martínez, On a paper of Edmunds and Opic on limiting interpolation of compact operators between $L_{p}$ spaces, Math. Nachr. 288 (2015), 167-175.

[14] F. Cobos, L.M. Fernández-Cabrera and A. Martínez, Measure of non-compactness of operators interpolated by limiting real methods, in Operator Theory: Advances and Applications, 219, Springer, Basel 2012, pp. 37-54.

[15] F. Cobos, L.M. Fernández-Cabrera and A. Martínez, Estimates for the spectrum on logarithmic interpolation spaces, J. Math. Anal. Appl. 437 (2016), 292-309.

[16] F. Cobos, L.M. Fernández-Cabrera, T. Kühn and T. Ullrich, On an extreme class of real interpolation spaces, J. Funct. Anal. 256 (2009), 2321-2366.

[17] F. Cobos, P. Fernández-Martínez and A. Martínez, Interpolation of the measure of non-compactnes by the real method, Studia Math. 135 (1999), 25-38.

[18] F. Cobos, T. Kühn and T. Schonbek, One-sided compactness results for Aronszajn-Gagliardo functors, J. Funct. Anal. 106 (1992), 274-313.

[19] F. Cobos and J. Peetre, Interpolation of compactness using Aronszajn-Gagliardo functors, Israel J. Math. 68 (1989), 220-240.

[20] F. Cobos and A. Segurado, Description of logarithmic interpolation spaces by means of the $J$ functional and applications, J. Funct. Anal. 268 (2015), 2906-2945.

[21] A. Connes, Noncommutative Geometry, Academic Press, San Diego, 1994.

[22] J.M. Cordeiro, Interpolación de Ciertas Clases de Operadores por Métodos Multidimensionales, $\mathrm{Ph}$. D. thesis, Publicaciones del Depto. de Matemática Aplicada, Universidad de Vigo, 1999. 
[23] M. Cwikel, Real and complex interpolation and extrapolation of compact operators, Duke Math. J. 65 (1992), 333-343.

[24] R.Ya. Doktorskii, Reiteration relations of the real interpolation method, Soviet Math. Dokl. 44 (1992), 665-669.

[25] D.E. Edmunds and W.D. Evans, Hardy Operators, Function Spaces and Embeddings, Springer, Berlin 2004.

[26] D.E. Edmunds and Yu. Netrusov, Entropy numbers and interpolation, Math. Ann. 351 (2011), 963-977.

[27] D.E. Edmunds and Yu. Netrusov, Entropy numbers of operators acting between vector-valued sequence spaces, Math. Nachr. 286 (2013), 614-630.

[28] D.E. Edmunds and B. Opic, Limiting variants of Krasnoselski1̌'s compact interpolation theorem, $J$. Funct. Anal. 266 (2014), 3265-3285.

[29] W.D. Evans and B. Opic, Real interpolation with logarithmic functors and reiteration, Canad. J. Math. 52 (2000), 920-960.

[30] W.D. Evans, B. Opic and L. Pick, Real interpolation with logarithmic functors, J. Inequal. Appl. 7 (2002), 187-269.

[31] J. Gustavsson, A function parameter in connection with interpolation of Banach spaces, Math. Scand. 42 (1978), 289-305.

[32] H. König, Eigenvalue Distribution of Compact Operators, Birkhäuser, Basel, 1986.

[33] G. Köthe, Topological Vector Spaces I, Springer, Berlin, 1969.

[34] M.A. Krasnosel'skiǐ, On a theorem of M. Riesz, Dokl. Akad. Nauk SSSR. 131 (1960), 246-248.

[35] J.L. Lions and J. Peetre, Sur une classe d'espaces d'interpolation, Publ. Math. Inst. Hautes Etudes Sci. 19 (1964), 5-68.

[36] M. Mastyło and E.B. Silva, Interpolation of the measure of non-compactness of bilinear operators, Trans. Amer. Math. Soc. 370 (2018), 8979-8997.

[37] P. Nilsson, Reiteration theorems for real interpolation and approximation spaces, Ann. Mat. Pura Appl. 132 (1982), 291-330.

[38] B. Opic and L. Pick, On generalized Lorentz-Zygmund spaces, Math. Inequal. Appl. 2 (1999), 391467.

[39] L.E. Persson, Interpolation with a parameter function, Math. Scand. 59 (1986), 199-222.

[40] M. Schechter, Principles of Functional Analysis, Amer. Math. Soc., Providence, 2001.

[41] R. Szwedek, Measure of non-compactness of operators interpolated by the real method, Studia Math. 175 (2006), 157-174.

[42] R. Szwedek, On interpolation of the measure of non-compactness by the complex method, Quart. J. Math. Oxford 66 (2015), 323-332.

[43] R. Szwedek, Geometric interpolation of entropy numbers, Quart. J. Math. Oxford 69 (2018), 377389.

[44] M.F. Teixeira and D.E. Edmunds, Interpolation theory and measures of non-compactness, Math. Nachr. 104 (1981), 129-135.

[45] H. Triebel, Interpolation Theory, Function Spaces, Differential Operators, North-Holland, Amsterdam, 1978.

[46] H. Triebel, Theory of Function Spaces II, Birkhäuser, Basel, 1992. 